Tables des nombres de classes et unités des corps quintiques cycliques de conducteur $\mathrm{f} \leq 10000$ 


\title{
Tables des nombres de classes et unités des corps quintiques cycliques de conducteur $f \leq 10000$
}

\author{
S.Jeannin
}

\section{Identification des corps étudiés}

Soit $K / \mathbb{Q}$ une extension cyclique de degré $l=5$, de conducteur $f$ et de groupe de Galois $G=<\sigma>$.

On sait que $\operatorname{Gal}\left(\mathbb{Q}\left(\mu_{f}\right) / \mathbb{Q}\right)$ est canoniquement isomorphe à $(\mathbb{Z} / f \mathbb{Z})^{\times} ;$on note $H$ le sous-groupe de $(\mathbb{Z} / f \mathbb{Z})^{\times}$, image de $\operatorname{Gal}\left(\mathbb{Q}\left(\mu_{f}\right) / K\right)$ par cet isomorphisme ( $H$ est appelé le groupe d'Artin de $K$ ), et soit $H^{+}$un système exact de représentants impairs de $H /\{+1,-1\}$. On pose :

$$
f=\prod_{i=1}^{k} p_{i}^{n_{i}}
$$

$p_{i}$ premiers distincts, où $n_{i}=1$ si $p_{i} \neq l$ et $n_{i}=2$ si $p_{i}=l$. Il est clair que $p_{i}=l$ ou $p_{i} \equiv 1(l)$, et que tout $p_{i}$ est alors totalement ramifié dans $K$. Il existe donc, pour $f$ donné, $(l-1)^{k-1}$ corps cycliques de degré premier impair $l$ et de conducteur $f$.

Soit $\chi$ un caractère associé à $K\left(\chi:(\mathbb{Z} / f \mathbb{Z})^{\times} \longrightarrow \mathbb{C}^{\times}\right.$, dont le noyau est $H)$. On sait que l'ensemble $\left\{\chi^{a}, a \bmod l,(a, l)=1\right\}$ caractérise $K$. On a

$$
(\mathbb{Z} / f \mathbb{Z})^{\times} \simeq \prod_{i=1}^{k}\left(\mathbb{Z} / p_{i}^{n_{i}} \mathbb{Z}\right)^{\times},
$$

il s'ensuit, en désignant par $(\mathbb{Z} / q \mathbb{Z})^{\times \perp}$ le groupe des caractères de $(\mathbb{Z} / q \mathbb{Z})^{\times}$, l'isomorphisme canonique:

$$
(\mathbb{Z} / f \mathbb{Z})^{\times \perp} \simeq \prod_{i=1}^{k}\left(\mathbb{Z} / p_{i}^{n_{i}} \mathbb{Z}\right)^{\times \perp} .
$$


On peut ainsi écrire tout caractère $\chi$ de $(\mathbb{Z} / f \mathbb{Z})^{\times}$sous la forme $\chi=\left(\ldots, \chi_{i}, \ldots\right)$, $\chi_{i} \in\left(\mathbb{Z} / p_{i}^{n_{i}} \mathbb{Z}\right)^{\times \perp}, 1 \leq i \leq k$. On précise ces isomorphismes en écrivant tout d'abord:

$$
(\mathbb{Z} / f \mathbb{Z})^{\times}=\bigoplus_{i=1}^{k} G_{i},
$$

où les $G_{i}$ correspondent aux sous-groupes $\operatorname{Gal}\left(\mathbb{Q}\left(\mu_{f}\right) / \mathbb{Q}\left(\mu_{f_{i}}\right)\right) \simeq \operatorname{Gal}\left(\mathbb{Q}\left(\mu_{f_{i}}\right) / \mathbb{Q}\right)$, en notant $f_{i}=p_{i}^{n_{i}}$ et $\vec{f}_{i}=f / f_{i}$ pour tout $p_{i}$ divisant $f$; on a alors $G_{i}=\{a \in$ $\left.(\mathbb{Z} / f \mathbb{Z})^{\times}, a \equiv 1\left(\bar{f}_{i}\right)\right\}$. De même:

$$
(\mathbb{Z} / f \mathbb{Z})^{\times \perp}=\bigoplus_{i=1}^{k} G_{i}^{\perp},
$$

où les éléments de $G_{i}^{\perp}$ (notés encore $\left.\chi_{i}\right)$ sont les caractères de $\mathbb{Q}\left(\mu_{f_{i}}\right)$, et tout caractère $\chi$ de $(\mathbb{Z} / f \mathbb{Z})^{\times}$s'écrit de façon unique:

$$
\chi=\prod_{i=1}^{k} \chi_{i}, \chi_{i} \in G_{i}^{\perp} .
$$

Soient maintenant $g_{1}, \ldots, g_{k} \in(\mathbb{Z} / f \mathbb{Z})^{\times}$des générateurs respectifs des sousgroupes $G_{i}$, et soit $\zeta_{l}$ une racine primitive $l$-ième de l'unité; définissons, pour chaque $i, 1 \leq i \leq k$, un caractère $\chi_{i}$ d'ordre $l$ de $G_{i}$ en posant:

$$
\begin{aligned}
& \chi_{i}\left(g_{i}\right)=\zeta_{l}, \\
& \chi_{i}\left(g_{j}\right)=1, \forall j \neq i .
\end{aligned}
$$

On définit alors un corps cyclique $K$, de degré premier impair $l$ et de conducteur $f$, par un caractère dont le noyau est $H$ en posant:

$$
\chi=\prod_{i=1}^{k} \chi_{i}^{a_{i}}, a_{i} \in\{1, \ldots, l-1\} .
$$

On remarque que si le $k$-uple $\left(a_{1}, \ldots, a_{k}\right)$ correspond à $K$, le $k$-uple $\left(\lambda a_{1}, \ldots, \lambda a_{k}\right)$ mod $l$, où $\lambda$ est un entier rationnel non nul modulo $l$, correspond lui-aussi à $K$.

\section{Rappel sur les unités des corps abéliens}

La méthode de dévissage de l'unité cyclotomique utilise le fait que $E$ est un $\mathbb{Z}\left[\mu_{l}\right]$-module sans torsion de rang 1 et que $F$ en est un sous- $\mathbb{Z}\left[\mu_{l}\right]-$ module libre. En effet, on a:

$$
F=\langle\eta\rangle_{\mathbf{z}\left[\mu_{1}\right]},
$$


où $\eta$ est l'unité cyclotomique génératrice de $F$ que l'on obtient comme suit:

Soient $\zeta=\exp (2 i \pi / f), \zeta^{\prime}=\exp (i \pi / f)$ et $\sigma_{g}$ un automorphisme de $\mathbb{Q}\left(\mu_{f}\right)$ défini par $\sigma_{g}: \zeta \longrightarrow \zeta^{g}$ tel que $\sigma_{g}$ restreint à $K$ donne $\sigma$. Puisque $f$ est impair et que $g$ est une classe modulo $f$, on peut choisir $g$ impair ( $g$ ou $g+f$ est impair) afin de préserver la signature de $\eta$ qui est connue grâce au lemme suivant (cf. [G]) :

Lemme 2.1 On $a \eta= \pm \prod_{x \in H^{+}} \frac{\sin (x \pi / f)}{\sin (x g \pi / f)}$

\section{Tables des corps quintiques cycliques de conducteur $f \leq 10000$}

Le programme mis au point, permettant de calculer le nombre de classes et les unités de tous les corps cycliques de degré $l=5$, utilise la méthode de dévissage de l'unité cyclotomique et reprend à sa base l'algorithme GALCYCL élaboré par G.Gras dans [GG].

La quatrième colonne de la table suivante donne le $k$-uple $\left(a_{1}, \ldots, a_{k}\right)$ (correspondant au corps $K$ que l'on étudie) où l'un des $a_{i}, 1 \leq i \leq k$, est égal à 1 .

La cinquième colonne donne l'entier gen $\in(\mathbb{Z} / f \mathbb{Z})^{\times}$dont le Frobenius est générateur de $\operatorname{Gal}(K / \mathbb{Q})$. La table donne le polynôme $p(x)$ d'une unité fondamentale génératrice de $E$ (vu comme $\mathbb{Z}\left[\mu_{l}\right]$-module) dans le cas où $K$ n'est pas principal. Dans le cas contraire l'unité fondamentale génératrice de $E$ est l'unité cyclotomique $\eta$. 


\begin{tabular}{|c|c|c|c|c|c|}
\hline$f$ & $k$ & $\left(g_{i}\right)_{i=1, \ldots, k}$ & $k-$ uple & gen & $h$ \\
\hline \multicolumn{6}{|c|}{$p(x)$} \\
\hline 25 & 1 & 2 & 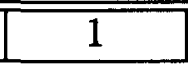 & 2 & 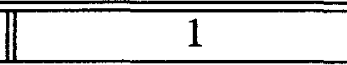 \\
\hline 11 & 1 & 2 & 1 & 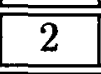 & 1 \\
\hline$\overline{31}$ & 11 & $\overline{3}$ & 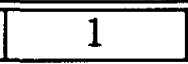 & $\overline{3}$ & 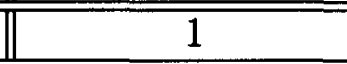 \\
\hline 41 & 11 & (26 & 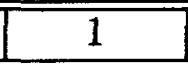 & 6 & 1 \\
\hline$\overline{61}$ & 1 & $\overline{2}$ & $\overline{1}$ & $\overline{2}$ & 1 \\
\hline$\overline{71}$ & 1 & $\overline{7}$ & 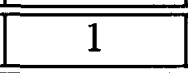 & $\overline{7}$ & 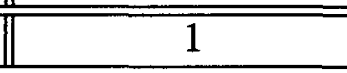 \\
\hline 101 & 1 & $\overline{22}$ & 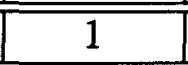 & 2 & $\overline{1}$ \\
\hline 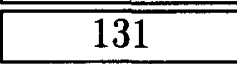 & 11 & $\overline{2}$ & $\overline{1}$ & 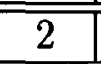 & 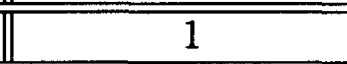 \\
\hline$\overline{151}$ & 1 & $\overline{\overline{2}}$ & $\overline{11}$ & $\overline{\overline{2}}$ & $\overline{\overline{1}}$ \\
\hline$\overline{181}$ & $1 \mid$ & $\overline{2}$ & 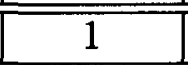 & $\overline{2}$ & 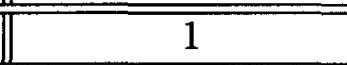 \\
\hline 191 & 11 & 19 & $\overline{1}$ & $\overline{\overline{19}}$ & $\overline{11}$ \\
\hline \multicolumn{6}{|c|}{$x^{5}+54 x^{4}+135 x^{3}-70 x^{2}+4 x+1$} \\
\hline 211 & 11 & 2 & 1 & 2 & 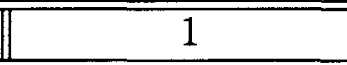 \\
\hline$\overline{241}$ & $1 \mid$ & 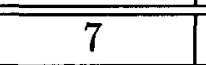 & 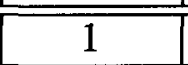 & 7 & 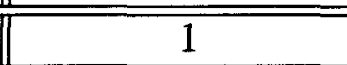 \\
\hline 251 & 11 & $\overline{6}$ & 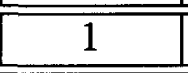 & 6 & 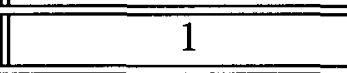 \\
\hline 271 & 11 & 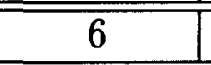 & $\overline{1}$ & 6 & 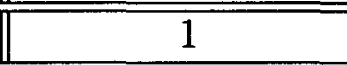 \\
\hline $275=25.11$ & 2 & 12,51 & $\overline{(1,1)}$ & 12 & $\overline{5}$ \\
\hline \multicolumn{6}{|c|}{$x^{5}-70 x^{4}+695 x^{3}+1050 x^{2}+240 x-1$} \\
\hline $275=25.11$ & 2 & 12,51 & $\overline{(2,1)}$ & 12 & 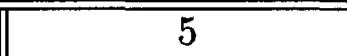 \\
\hline \multicolumn{6}{|c|}{$x^{5}-6120 x^{4}-157705 x^{3}+729250 x^{2}-6360 x-1$} \\
\hline $275=25.11$ & 2 & 12,51 & $\overline{(3,1)}$ & 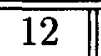 & $\overline{5}$ \\
\hline \multicolumn{6}{|c|}{$x^{5}-345 x^{4}-121130 x^{3}-3395750 x^{2}+71465 x-1$} \\
\hline $275=25.11$ & 2 & 12,51 & $\overline{(4,1)}$ & 12 & 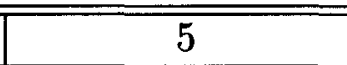 \\
\hline \multicolumn{6}{|c|}{$x^{5}-70 x^{4}+145 x^{3}+225 x^{2}-35 x-1$} \\
\hline 281 & 11 & 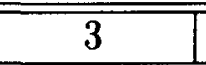 & 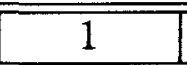 & $\overline{3}$ & 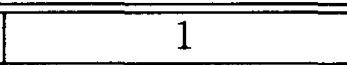 \\
\hline 311 & $1 \mid$ & $\overline{177}$ & 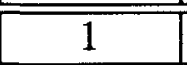 & 17 & $\overline{1} 1$ \\
\hline 331 & 1 & 3 & 1 & 3 & 1 \\
\hline $341=11.31$ & 2 & 63,12 & $\overline{(1,1)}$ & $\overline{63}$ & $\overline{5}$ \\
\hline \multicolumn{6}{|c|}{$x^{5}+1107 x^{4}-177703 x^{3}-1723574 x^{2}+5817 x-1$} \\
\hline $341=11.31$ & $2 \mid$ & 63,12 & $(2,1)$ & "63 & 5 \\
\hline \multicolumn{6}{|c|}{$x^{5}+11027683 x^{4}-234041982 x^{3}-186636962 x^{2}-172867 x-1$} \\
\hline $341=11.31$ & $2 \mid$ & 63,12 & $\overline{(3,1)}$ & 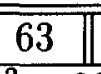 & 5 \\
\hline
\end{tabular}




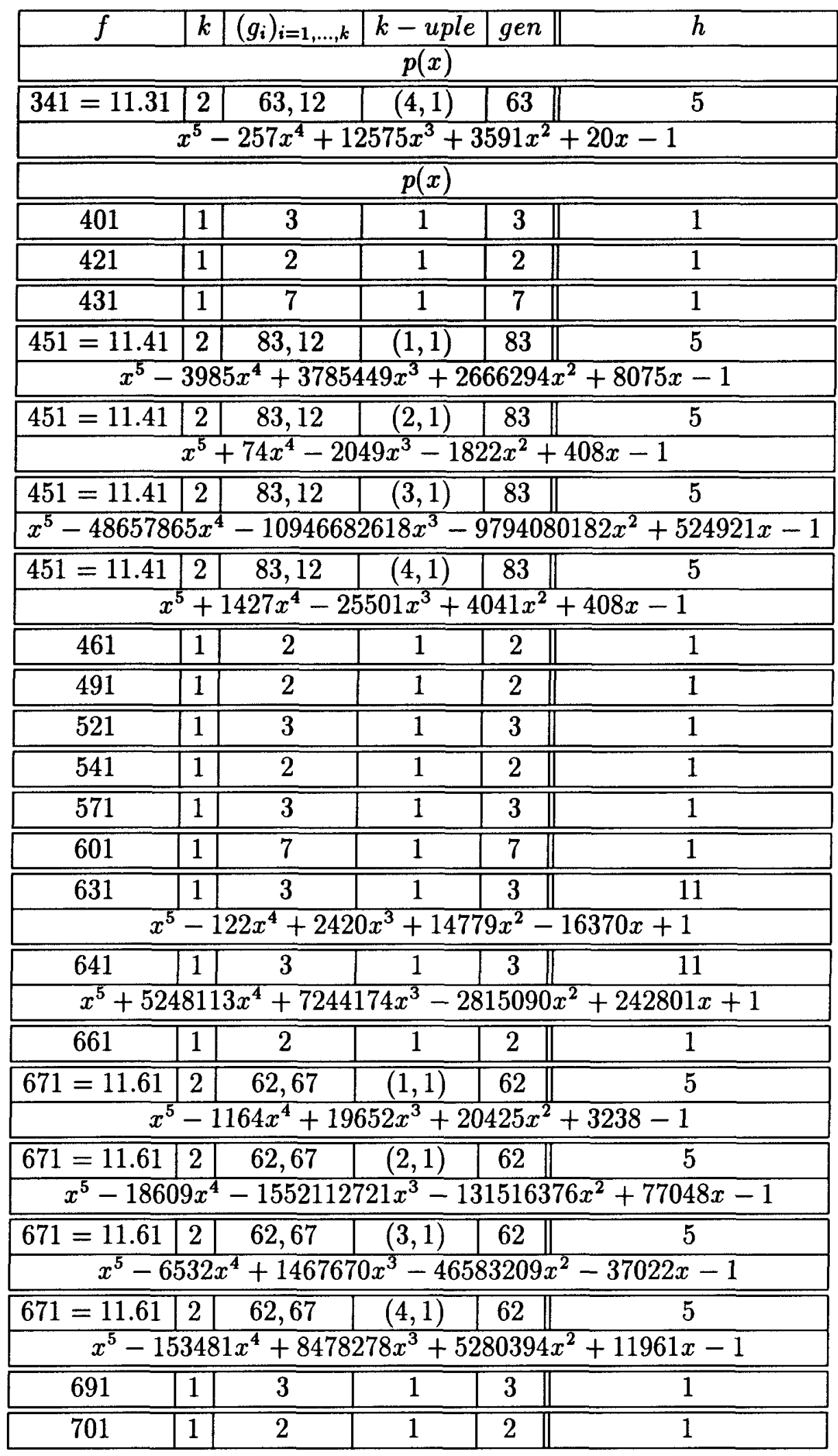




\begin{tabular}{|c|c|c|c|c|c|}
\hline$f$ & $k$ & $\left(g_{i}\right)_{i=1, \ldots, k}$ & $k-$ uple & gen & $\bar{h}$ \\
\hline \multicolumn{6}{|c|}{$p(x)$} \\
\hline 751 & 1 & 3 & 1 & 3 & 1 \\
\hline 761 & 1 & 6 & 1 & 6 & 1 \\
\hline $775=25.31$ & 2 & 63,176 & $(1,1)$ & 63 & 5 \\
\hline \multicolumn{6}{|c|}{$\begin{aligned} x^{5}+26706063515 x^{4} & +28012225465930 x^{3}+796319168710 x^{2} \\
& +108968725 x-1\end{aligned}$} \\
\hline $775=25.31$ & 2 & 63,176 & $\overline{(2,1)}$ & 63 & $\overline{5}$ \\
\hline \multicolumn{6}{|c|}{$x^{5}-4364960 x^{4}+25055289505 x^{3}+79000235 x^{2}+50225 x-1$} \\
\hline $775=25.31$ & 2 & $\overline{63,176}$ & $\overline{(3,1)}$ & $\overline{63}$ & $\overline{5}$ \\
\hline \multicolumn{6}{|c|}{$x^{5}-160 x^{4}-1695 x^{3}-3265 x^{2}+625 x-1$} \\
\hline $775=25.31$ & 2 & 63,176 & $(4,1)$ & 63 & 5 \\
\hline \multicolumn{6}{|c|}{$x^{5}-4810 x^{4}+24655 x^{3}+23860 x^{2}-1700 x-1$} \\
\hline $781=11.71$ & 2 & 72,56 & $(1,1)$ & 72 & 5 \\
\hline \multicolumn{6}{|c|}{$x^{5}+22453 x^{4}-5796367 x^{3}-174000 x^{2}+2897 x-1$} \\
\hline $781=11.71$ & 2 & 72,56 & $(2,1)$ & 72 & 5 \\
\hline \multicolumn{6}{|c|}{$x^{5}-196 x^{4}-6814 x^{3}-54507 x^{2}+3678 x-1$} \\
\hline $781=11.71$ & 2 & $\overline{72,56}$ & $(3,1)$ & 72 & 5 \\
\hline \multicolumn{6}{|c|}{$x^{5}+331349207 x^{4}+34066434529 x^{3}-7775368735 x^{2}-232184 x-$} \\
\hline $781=11.71$ & 2 & $\overline{72,56}$ & $(4,1)$ & 72 & 5 \\
\hline \multicolumn{6}{|c|}{$\begin{aligned} & x^{5}-3541848473 x^{4}+101569333718 x^{3}+7883591450 x^{2} \\
&+146601 x-1 \\
&\end{aligned}$} \\
\hline$\overline{811}$ & 1 & 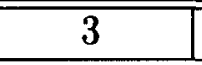 & 1 & 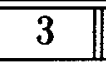 & 1 \\
\hline 821 & 1 & 2 & 1 & 2 & 1 \\
\hline 881 & 1 & 2 & 1 & 2 & 1 \\
\hline 911 & 1 & 17 & 1 & 17 & 1 \\
\hline 941 & 1 & 2 & 1 & 2 & 16 \\
\hline \multicolumn{6}{|c|}{$x^{5}-16 x^{4}-274 x^{3}-817 x^{2}+178 x-1$} \\
\hline 971 & 1 & 6 & 1 & 6 & 1 \\
\hline 991 & 1 & 6 & 1 & 6 & 1 \\
\hline 1021 & 1 & 10 & 1 & 10 & 1 \\
\hline $1025=25.41$ & 2 & 42,26 & $(1,1)$ & 42 & 5 \\
\hline \multicolumn{6}{|c|}{$\begin{aligned} & x^{5}-28087745 x^{4}+ 39271750754270 x^{3}+85442622850 x^{2} \\
&- 32530335 x-1 \\
&\end{aligned}$} \\
\hline $1025=25.41$ & 2 & 42,26 & $(2,1)$ & 42 & 5 \\
\hline \multicolumn{6}{|c|}{$x^{5}-1720 x^{4}+2970 x^{3}+1475 x^{2}+90 x-1$} \\
\hline $1025=25.41$ & 2 & 42,26 & $(3,1)$ & 42 & 5 \\
\hline & & $4=00470$ & $x-12$ & $x x-$ & -12. \\
\hline
\end{tabular}




\begin{tabular}{|c|c|c|c|c|c|}
\hline$f$ & $k$ & $\left(g_{i}\right)_{i=1, \ldots, k}$ & $k-u p l e$ & gen & $h$ \\
\hline \multicolumn{6}{|c|}{$p(x)$} \\
\hline $1025=25.41$ & 2 & 42,26 & $\overline{(4,1)}$ & $\overline{42}$ & 5 \\
\hline \multicolumn{6}{|c|}{$x^{5}-4795 x^{4}-16505 x^{3}-14925 x^{2}-4010 x-1$} \\
\hline 1031 & 11 & $\overline{14}$ & $\overline{11}$ & $\overline{14}$ & 1 \\
\hline 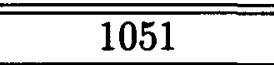 & 1 & $\overline{7}$ & 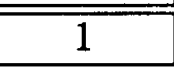 & $\overline{7}$ & $\overline{1} 1$ \\
\hline$\overline{\overline{1061}}$ & 11 & $\overline{2}$ & $\overline{1}$ & $\overline{2}$ & $\overline{11}$ \\
\hline 1091 & \begin{tabular}{|l|l}
1 \\
\end{tabular} & $\overline{\overline{2}}$ & $\overline{11}$ & $\overline{\overline{2}}$ & $\overline{11}$ \\
\hline $1111=11.101$ & 2 & $\overline{\overline{304,12}}$ & 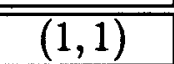 & 304 & $\overline{\overline{5}}$ \\
\hline \multicolumn{6}{|c|}{$x^{5}-21289 x^{4}+52956 x^{3}+45611 x^{2}-11079 x-1$} \\
\hline $1111=11.101$ & \begin{tabular}{l|l}
2 \\
\end{tabular} & 304,12 & $\overline{(2,1)}$ & 304 & 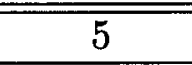 \\
\hline \multicolumn{6}{|c|}{$x^{5}-180 x^{4}-1483 x^{3}-1051 x^{2}+2253 x-1$} \\
\hline$\overline{1111}=11.101$ & \begin{tabular}{|l|l}
2 & \\
\end{tabular} & 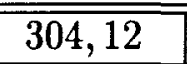 & $(3,1)$ & \begin{tabular}{|l||}
304 \\
\end{tabular} & $\overline{5}$ \\
\hline \multicolumn{6}{|c|}{$\begin{array}{c}x^{5}-508669137819 x^{4}+7215250189081002 x^{3} \\
+5532073174554118 x^{2}-27386004492299 x-1 \\
\end{array}$} \\
\hline $1111=11.101$ & 2 & $\overline{304,12}$ & $(4,1)$ & 304 & $\overline{5}$ \\
\hline \multicolumn{6}{|c|}{$\begin{array}{c}x^{5}+5879232 x^{4}-2017007840527 x^{3}+617196102055 x^{2} \\
-3271070607 x-1\end{array}$} \\
\hline 1151 & \begin{tabular}{|l|l}
1 & \\
\end{tabular} & 17 & 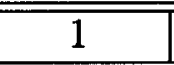 & 17 & 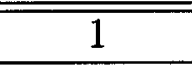 \\
\hline 1171 & 1 & 2 & $\overline{1}$ & $\overline{2}$ & 1 \\
\hline 1181 & $\overline{11}$ & $\overline{7}$ & $\overline{1}$ & $\overline{77}$ & $\overline{11}$ \\
\hline 1201 & 1 & 11 & 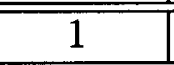 & 11 & 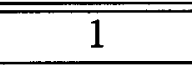 \\
\hline$\overline{1231}$ & 1 & $\overline{\overline{3}}$ & $\overline{1}$ & $\overline{\overline{3}}$ & $\overline{11}$ \\
\hline $21271=31.41$ & \begin{tabular}{|l|l}
2 & \\
\end{tabular} & $\begin{array}{l}42,63 \\
\end{array}$ & $(1,1)$ & 42 & 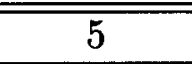 \\
\hline \multicolumn{6}{|c|}{$x^{5}-166550 x^{4}-106786426 x^{3}+6310661 x^{2}+41736 x-1$} \\
\hline $1271=31.41$ & \begin{tabular}{l|l}
2 & \\
\end{tabular} & 42,63 & $(2,1)$ & 42 & 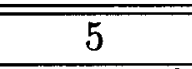 \\
\hline \multicolumn{6}{|c|}{$\begin{array}{c}x^{5}-653609257 x^{4}+120066881130950 x^{3}-40204709078 x^{2} \\
-579783 x-1\end{array}$} \\
\hline $1271=31.41$ & $2 \mid$ & 42,63 & $(3,1)$ & 42 & 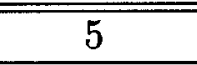 \\
\hline \multicolumn{6}{|c|}{$x^{5}-1320 x^{4}-105041 x^{3}+57993334 x^{2}+16316 x-1$} \\
\hline $1271=31.41$ & \begin{tabular}{l|l}
2 & \\
\end{tabular} & 42,63 & $(4,1)$ & 42 & $\overline{55=5.11}$ \\
\hline \multicolumn{6}{|c|}{$x^{5}+73 x^{4}+1369 x^{3}+1685 x^{2}+226 x-1$} \\
\hline 1291 & 1 & 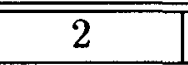 & 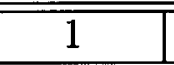 & 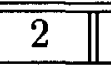 & $\overline{1.1}$ \\
\hline$\overline{1301}$ & 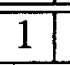 & 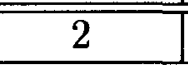 & 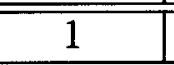 & 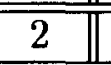 & 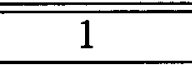 \\
\hline$\overline{1321}$ & $\bar{~} 1$ & $\overline{\overline{13}}$ & $\overline{1}$ & 13 & $\overline{1}$ \\
\hline 1361 & 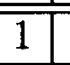 & 3 & $\overline{1}$ & 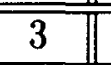 & $\bar{~} 1$ \\
\hline 1381 & $\overline{1}$ & $\overline{2}$ & 1 & $\overline{2}$ & $\overline{1}$ \\
\hline
\end{tabular}




\begin{tabular}{|c|c|c|c|c|c|}
\hline$f$ & & $\left(g_{i}\right)_{i=1,}$ & $k-$ uple & gen & $h$ \\
\hline \multicolumn{6}{|c|}{$p(x)$} \\
\hline $1441=11.131$ & 2 & $\overline{525,23}$ & $\overline{(1,1)}$ & \begin{tabular}{|l||}
525 \\
\end{tabular} & 5 \\
\hline \multicolumn{6}{|c|}{$x^{5}-144352620 x^{4}-231493813 x^{3}+153515176 x^{2}-15561618 x-1$} \\
\hline $1441=11.131$ & \begin{tabular}{|l|}
2 \\
\end{tabular} & 525,23 & $\overline{(2,1)}$ & \begin{tabular}{|l||}
525 \\
\end{tabular} & $\overline{5}$ \\
\hline \multicolumn{6}{|c|}{$x^{5}+7962521 x^{4}-138540667 x^{3}-95076057 x^{2}-145800 x-1$} \\
\hline $1441=11.131$ & \begin{tabular}{|l|}
2 \\
\end{tabular} & 525,23 & $\overline{(3,1)}$ & $\mid$\begin{tabular}{|l||}
525 \\
\end{tabular} & $\overline{55}$ \\
\hline \multicolumn{6}{|c|}{$x^{5}-4768 x^{4}-98033 x^{3}+395957 x^{2}-259 x-1$} \\
\hline $1441=11.131$ & 21 & $\overline{525,23}$ & $\overline{(4,1)}$ & 525 & 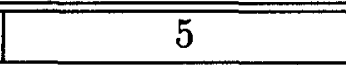 \\
\hline \multicolumn{6}{|c|}{$\begin{aligned} x^{5}+568491347 x^{4}+ & 65525680949266 x^{3}-686339909858514 x^{2} \\
& -9528053651 x-1\end{aligned}$} \\
\hline 1451 & 11 & 2 & 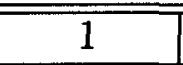 & 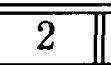 & 1 \\
\hline 1471 & 1 & $\underline{\mathbf{b}}$ & 1 & 6 & 1 \\
\hline 1481 & 1 & 3 & $\overline{11}$ & $\overline{\overline{3}}$ & $\overline{11}$ \\
\hline 1511 & 1 & 11 & 1 & 11 & 1 \\
\hline $1525=25.61$ & \begin{tabular}{l|l}
2 & \\
\end{tabular} & $\overline{62,26}$ & $\overline{(1,1)}$ & $\overline{62}$ & 5 \\
\hline \multicolumn{6}{|c|}{$\begin{array}{c}x^{5}-3760264465 x^{4}+203199995150 x^{3}-65855949870 x^{2} \\
-2235855 x-1 \\
\end{array}$} \\
\hline $1525=25.61$ & \begin{tabular}{l|l}
2 \\
\end{tabular} & 62,26 & $(2,1)$ & 62 & 5 \\
\hline \multicolumn{6}{|c|}{$x^{5}-17065 x^{4}+4725150 x^{3}-83370870 x^{2}-210655 x-1$} \\
\hline $1525=25.61$ & \begin{tabular}{l|l}
2 & \\
\end{tabular} & 62,26 & $(3,1)$ & 62 & $\overline{5}$ \\
\hline \multicolumn{6}{|c|}{$x^{5}+3453835 x^{4}-219045725 x^{3}-92604745 x^{2}+59270 x-1$} \\
\hline $1525=25.61$ & \begin{tabular}{l|l}
2 & \\
\end{tabular} & 62,26 & $\begin{array}{l}(4,1) \\
\end{array}$ & $\bar{~} 62$ & $\overline{5}$ \\
\hline \multicolumn{6}{|c|}{$x^{5}-14015 x^{4}-182067525 x^{3}+1028294655 x^{2}+446620 x-1$} \\
\hline 1511 & 11 & 11 & 1 & 11 & 1 \\
\hline 1531 & 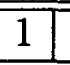 & $\overline{2}$ & 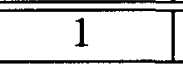 & 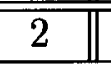 & 1 \\
\hline 1571 & 1 & 2 & 1 & 2 & 1 \\
\hline 1601 & 1 & 3 & 1 & 3 & 1 \\
\hline 1621 & 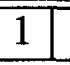 & 2 & 1 & 2 & $\overline{1}$ \\
\hline $1661=11.151$ & $2 \mid$ & $\begin{array}{l}303,12 \\
\end{array}$ & $(1,1)$ & 303 & 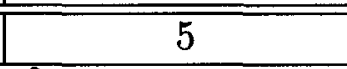 \\
\hline \multicolumn{6}{|c|}{$x^{5}-18868 x^{4}-3026338296 x^{3}-2918459485 x^{2}+153132 x-1$} \\
\hline $1661=11.151$ & $2 \mid$ & 303,12 & $(2,1)$ & 303|| & 5 \\
\hline \multicolumn{6}{|c|}{$x^{5}+868106 x^{4}+280503418 x^{3}-1759613005 x^{2}-2268663242 x-1$} \\
\hline $1661=11.151$ & $2 \mid$ & 303,12 & $(3,1)$ & 303 & 5 \\
\hline \multicolumn{6}{|c|}{$x^{5}+1064 x^{4}-971303 x^{3}-23265062 x^{2}-2464604 x-1$} \\
\hline $1661=11.151$ & 2 & 303,12 & $\overline{(4,1)}$ & 303 & 5 \\
\hline & & & 21 & $9 x^{2}-$ & $1 x-$ \\
\hline
\end{tabular}




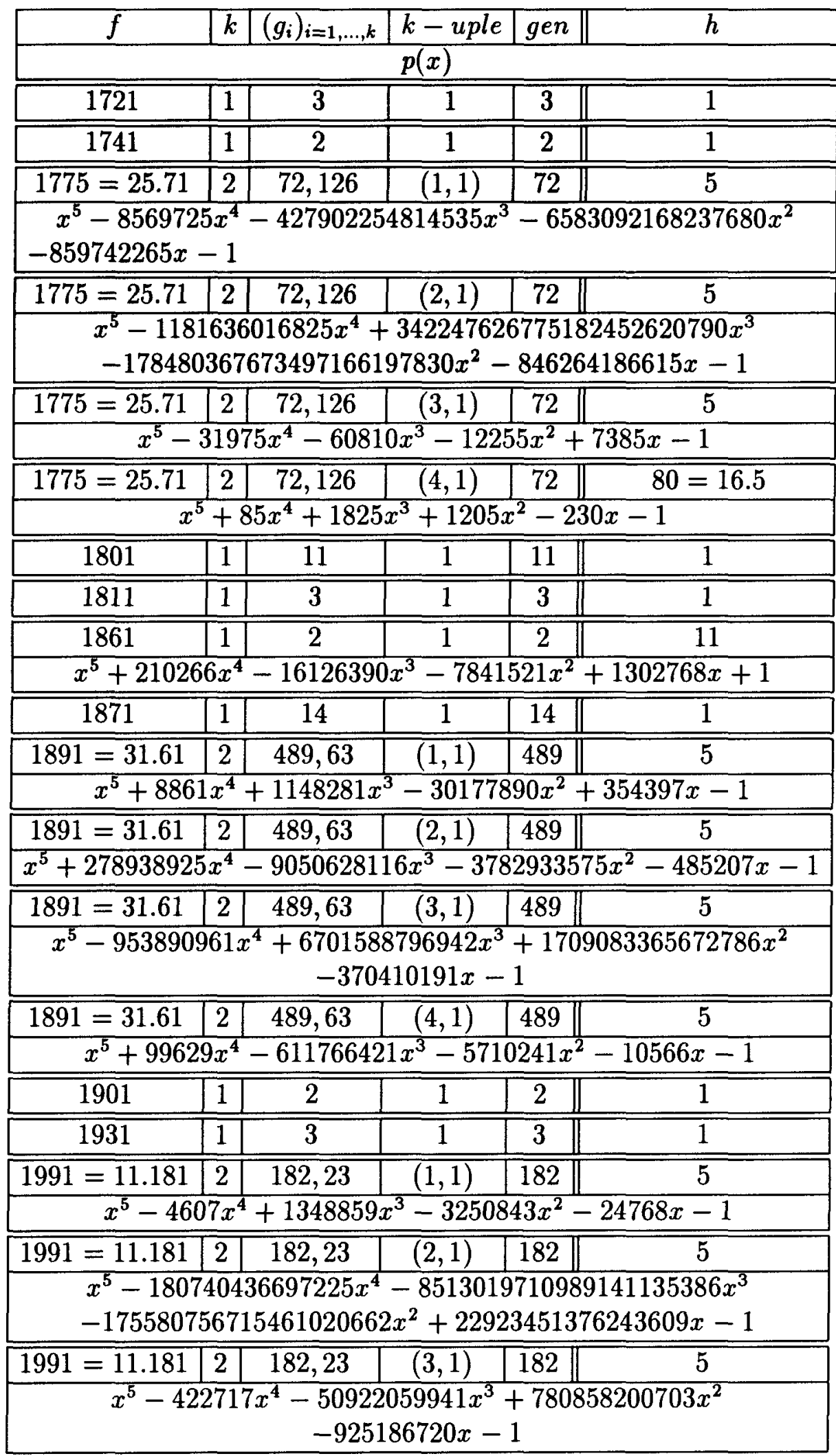




\begin{tabular}{|c|c|c|c|c|c|}
\hline$f$ & $k$ & $\left(g_{i}\right)_{i=1, \ldots, k}$ & $k$-uple & gen & $h$ \\
\hline \multicolumn{6}{|c|}{$p(x)$} \\
\hline $1991=11.181$ & 2 & 182,23 & $(4,1)$ & 182 & 5 \\
\hline \multicolumn{6}{|c|}{$\begin{array}{c}x^{5}-376924 x^{4}-7932961349 x^{3}+41644728068 x^{2} \\
+3651381628 x-1\end{array}$} \\
\hline 2011 & 1 & 3 & 1 & 3 & 1 \\
\hline 2081 & 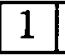 & 3 & 1 & 3 & 1 \\
\hline $2101=11.191$ & 2 & 574,56 & $(1,1)$ & 574 & 5 \\
\hline \multicolumn{6}{|c|}{$\begin{array}{c}x^{5}+181240850 x^{4}-64848013817 x^{3}-482984839477 x^{2} \\
-16573829 x-1\end{array}$} \\
\hline $2101=11.191$ & 2 & 574,56 & $(2,1)$ & 574 & 5 \\
\hline \multicolumn{6}{|c|}{$\begin{array}{c}x^{5}-36290352209 x^{4}-6334327661842 x^{3}-4787458620590 x^{2} \\
+2294805210129 x-1\end{array}$} \\
\hline $2101=11.191$ & 2 & 574,56 & $(3,1)$ & $\overline{574}$ & 5 \\
\hline \multicolumn{6}{|c|}{$x^{5}+4388 x^{4}-5271017 x^{3}+8575317 x^{2}-690269 x-1$} \\
\hline $2101=11.191$ & 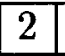 & 574,56 & $(4,1)$ & $\overline{574}$ & $\overline{5}$ \\
\hline \multicolumn{6}{|c|}{$x^{5}+149357 x^{4}+94188222 x^{3}-36764263 x^{2}-110393 x-1$} \\
\hline 2111 & 1 & 7 & 1 & 7 & 1 \\
\hline 2131 & \begin{tabular}{|l|l}
1 & \\
\end{tabular} & 2 & 1 & $\overline{2}$ & 1 \\
\hline 2141 & \begin{tabular}{|l|l|}
1 & \\
\end{tabular} & 2 & 1 & 2 & 1 \\
\hline 2161 & \begin{tabular}{|l|l|}
1 & \\
\end{tabular} & 23 & 1 & 23 & 16 \\
\hline \multicolumn{6}{|c|}{$\begin{aligned} & x^{5}+38708510 x^{4}-7787862611 x^{3}-653234794177 x^{2} \\
&+526865209 x-1 \\
&\end{aligned}$} \\
\hline $2201=31.71$ & \begin{tabular}{l|l}
2 & \\
\end{tabular} & 427,63 & $(1,1)$ & 427 & 5 \\
\hline \multicolumn{6}{|c|}{$\begin{array}{c}x^{5}-4875046816020033 x^{4}-26095845046769378 x^{3} \\
-64598151994046 x^{2}+16087521 x-1 \\
\end{array}$} \\
\hline $2201=31.71$ & \begin{tabular}{l|l}
2 & \\
\end{tabular} & 427,63 & $(2,1)$ & 427 & 5 \\
\hline \multicolumn{6}{|c|}{$x^{5}-49186 x^{4}+13484819 x^{3}+8585554 x^{2}-17196 x-1$} \\
\hline $2201=31.71$ & \begin{tabular}{|l|l|}
2 & \\
\end{tabular} & 427,63 & $(3,1)$ & 427 & 5 \\
\hline \multicolumn{6}{|c|}{$\begin{aligned} x^{5}+5945351441 x^{4}- & 887663164246 x^{3}+-156477347605 x^{2} \\
& +13551969 x-1\end{aligned}$} \\
\hline $2201=31.71$ & $2 \mid$ & 427,63 & $(4,1)$ & 427 & 5 \\
\hline$x^{5}+195125 x^{4}$ & +49 & $2877921 x^{3}$ & +217371 & $3656 x^{2}$ & $-257105 x-1$ \\
\hline 2221 & \begin{tabular}{l|l}
1 & \\
\end{tabular} & 2 & 1 & 2 & 1 \\
\hline 2251 & $1 \mid$ & 7 & 1 & 7 & 1 \\
\hline 2281 & 11 & 7 & 1 & 7 & 1 \\
\hline
\end{tabular}




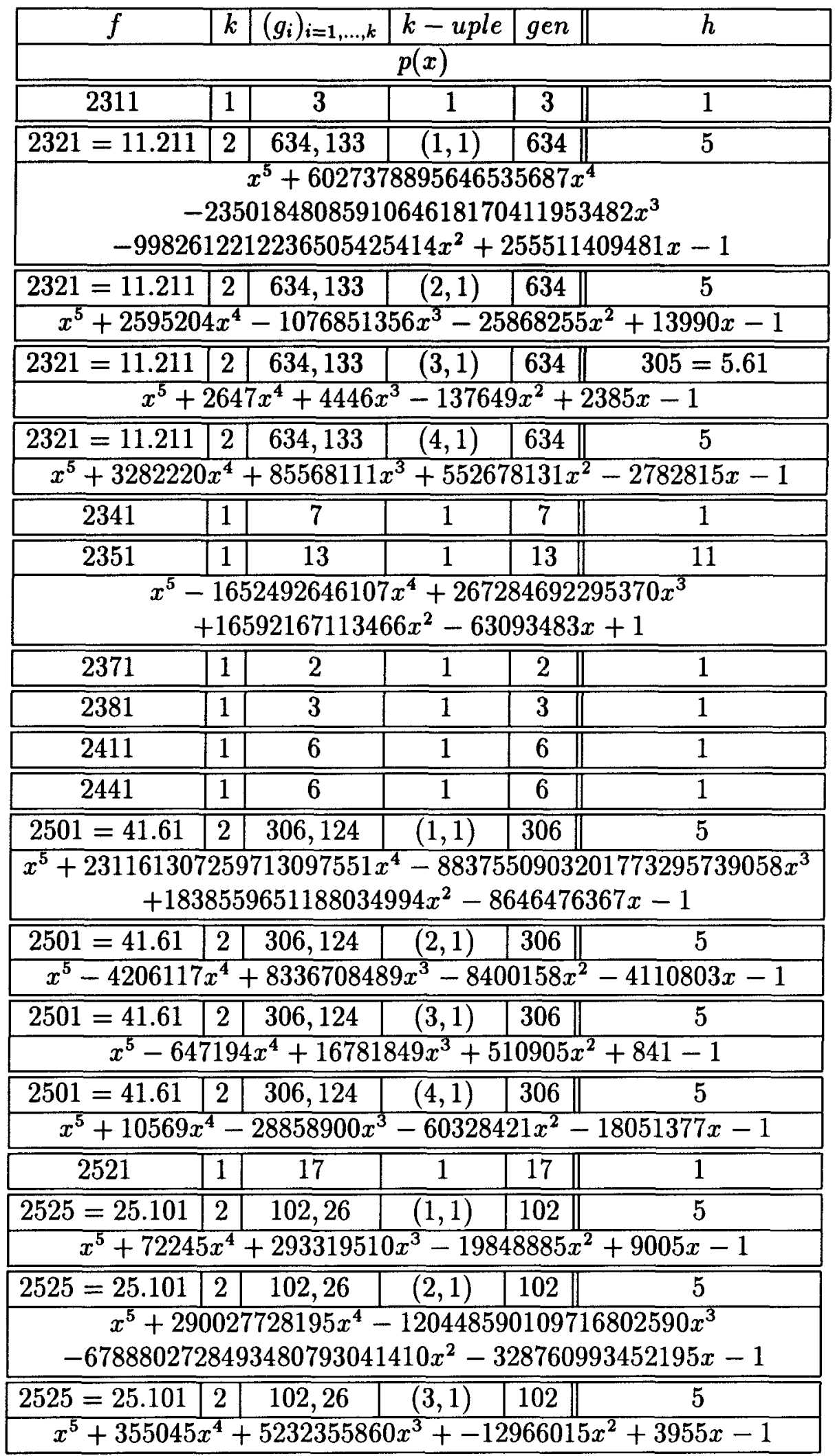




\begin{tabular}{|c|c|c|c|c|c|}
\hline$f$ & $k$ & $\left(g_{i}\right)_{i=1, \ldots, k}$ & $k-u p l e$ & gen & $h$ \\
\hline \multicolumn{6}{|c|}{$p(x)$} \\
\hline $2525=25.101$ & 2 & $\bar{~} 102,26$ & $\overline{(4,1)}$ & 102 & $\overline{\overline{5}}$ \\
\hline \multicolumn{6}{|c|}{$\begin{array}{c}x^{5}-602034205 x^{4}-865464761749265 x^{3} \\
+72933833246169109640 x^{2}-26971465295 x-1\end{array}$} \\
\hline $2 \overline{2531}$ & \begin{tabular}{l|l}
1 & \\
\end{tabular} & $\overline{22}$ & $\overline{1}$ & $\overline{2}$ & $\overline{11}$ \\
\hline$\overline{2551}$ & 1 & $\overline{\overline{6}}$ & 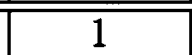 & 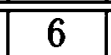 & $\overline{\overline{1}}$ \\
\hline 2591 & \begin{tabular}{l|l}
1 & 1 \\
\end{tabular} & 7 & 1 & 7 & 1 \\
\hline$\overline{\overline{2621}}$ & (1) & $\overline{\overline{2}}$ & $\overline{11}$ & $\overline{\overline{2}}$ & $\overline{11}$ \\
\hline $2651=11.241$ & 2 & 965,34 & $\overline{(1,1)}$ & 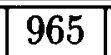 & $\overline{25}$ \\
\hline \multicolumn{6}{|c|}{$x^{5}+754751 x^{4}+3374405790 x^{3}-21527659765 x^{2}+348921 x-1$} \\
\hline $2651=11.241$ & 2 & 965,34 & $\overline{(2,1)}$ & \begin{tabular}{|c|}
965 \\
\end{tabular} & 25 \\
\hline \multicolumn{6}{|c|}{$x^{5}+191534 x^{4}-4409976873 x^{3}+2956837178 x^{2}+300480 x-1$} \\
\hline $2651=11.241$ & 2 & $\overline{965,34}$ & $\overline{(3,1)}$ & \begin{tabular}{|c||}
965 \\
\end{tabular} & 25 \\
\hline \multicolumn{6}{|c|}{$x^{5}-363730 x^{4}-5065056566 x^{3}-12782463279295 x^{2}-8838722 x-1$} \\
\hline $2651=11.241$ & 22 & $\overline{9965,34}$ & $\overline{(4,1)}$ & \begin{tabular}{|c||}
965 \\
\end{tabular} & 25 \\
\hline \multicolumn{6}{|c|}{$x^{5}+29766813 x^{4}+3594871144 x^{3}+13071196273 x^{2}+6461645-1$} \\
\hline 2671 & $1 \mid$ & 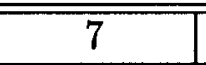 & $\overline{1.1}$ & 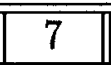 & 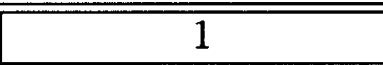 \\
\hline 2711 & 1 & 7 & $\overline{1}$ & $\overline{7}$ & $\overline{1}$ \\
\hline$\overline{2731}$ & (1) & 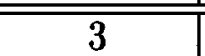 & $\bar{~} 1$ & 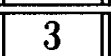 & $\overline{11}$ \\
\hline 2741 & 1 & $\overline{2}$ & $\overline{11}$ & $\overline{22}$ & $\overline{11}$ \\
\hline $2761=11.251$ & 2 & $\overline{503,34}$ & $(1,1)$ & 503 & 5 \\
\hline \multicolumn{6}{|c|}{$\begin{aligned} x^{5}+112145051 x^{4}+ & 859169632667458 x^{3}+1902742288811270 x^{2} \\
& +6940179462973 x-1\end{aligned}$} \\
\hline $2761=11.251$ & 2 & $\overline{5503,34}$ & (2,1) & $\mid$\begin{tabular}{|l||}
503 \\
\end{tabular} & $\overline{\overline{5}}$ \\
\hline \multicolumn{6}{|c|}{$\begin{array}{c}x^{5}-27213663 x^{4}+25888750181879 x^{3}-68200269923000 x^{2} \\
-16597033 x-1\end{array}$} \\
\hline $2761=11.251$ & 2 & $\overline{503,34}$ & $\overline{(\overline{(3,1)}}$ & \begin{tabular}{|l||}
503 \\
\end{tabular} & $\overline{\overline{5}}$ \\
\hline \multicolumn{6}{|c|}{$\begin{array}{c}x^{5}-36534799 x^{4}-2306557659371 x^{3}+2041004444110 x^{2} \\
-6105233 x-1 \\
\end{array}$} \\
\hline $2761=11.251$ & 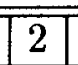 & 503,34 & \begin{tabular}{l|l|l}
$(4,1)$ \\
\end{tabular} & 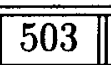 & 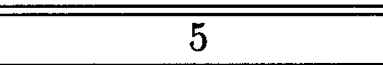 \\
\hline \multicolumn{6}{|c|}{$\begin{aligned} x^{5}-680453 x^{4}- & 477951616833 x^{3}+360647662578 x^{2} \\
& +179964079 x-1\end{aligned}$} \\
\hline 2801 & 11 & $\overline{\overline{3}}$ & $\overline{11}$ & 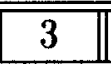 & $\overline{11}$ \\
\hline$\overline{2851}$ & $1 \mid$ & $\overline{2}$ & $\overline{1}$ & $\overline{2}$ & $\overline{1}$ \\
\hline 2861 & 1 & 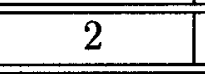 & 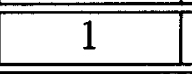 & 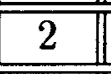 & 1 \\
\hline $2911=41.71$ & 2 & 356,42 & $\overline{(1,1)}$ & \begin{tabular}{|l||}
356 \\
\end{tabular} & $\overline{5}$ \\
\hline$x^{5}+1647905 x$ & & 38207421 & $8 x^{3}+9$ & & $x^{2}+138455 X$ \\
\hline
\end{tabular}




\begin{tabular}{|c|c|c|c|c|}
\hline f & \begin{tabular}{l|l}
$k$ & $\left(g_{i}\right)_{i=1, \ldots, k}$ \\
\end{tabular} & $k-$ uple & gen & $h$ \\
\hline \multicolumn{5}{|c|}{$p(x)$} \\
\hline $2911=41.71$ & \begin{tabular}{|l|r|}
2 & 356,42 \\
\end{tabular} & $(2,1)$ & 356 & 5 \\
\hline \multicolumn{5}{|c|}{$\begin{array}{c}x^{5}-71821204749 x^{4}-550245743776835 x^{3}-90456968638875 x^{2} \\
-5566016666 x-1\end{array}$} \\
\hline $2911=41.71$ & $\overline{356,42}$ & $\overline{(3,1)}$ & 356 & $\overline{5}$ \\
\hline \multicolumn{5}{|c|}{$\begin{array}{c}x^{5}-46744983165469 x^{4}-5003112041118396798 x^{3} \\
-40105059284898 x^{2}-14131267 x-1\end{array}$} \\
\hline $2911=41.71$ & $\overline{\overline{356,42}}$ & $(4,1)$ & 356 & 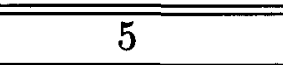 \\
\hline \multicolumn{5}{|c|}{$x^{5}+279 x^{4}-625003 x^{3}-8369348 x^{2}-3180085 x-1$} \\
\hline 2971 & 1 & 1 & 10 & 1 \\
\hline $2981=11.271$ & $\overline{272,133}$ & $\overline{(1,1)}$ & 272 & 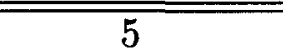 \\
\hline \multicolumn{5}{|c|}{$x^{5}-17236913 x^{4}-664958661 x^{3}-4396385133 x^{2}+18713214 x-1$} \\
\hline $2981=11.271$ & \begin{tabular}{|l|l|}
2 & 272,133 \\
\end{tabular} & $\overline{(2,1)}$ & 272 & $\overline{\overline{5}}$ \\
\hline \multicolumn{5}{|c|}{$\begin{array}{c}x^{5}-5106713118 x^{4}-357168814996 x^{3}-400874405137 x^{2} \\
+7641780 x-1 \\
\end{array}$} \\
\hline $2981=11.271$ & \begin{tabular}{|l|l|}
2 & 272,133 \\
\end{tabular} & $(3,1)$ & 272 & $\overline{5}$ \\
\hline \multicolumn{5}{|c|}{$\begin{array}{c}x^{5}-443966281186613377943903432017 x^{4} \\
-328729754465437081316888607802802 x^{3}+ \\
21564737806811672455220790014206802 x^{2} \\
-592940379849720844367 x-1\end{array}$} \\
\hline $2981=11.271$ & \begin{tabular}{|l|l|}
2 & 272,133 \\
\end{tabular} & $(4,1)$ & 272 & 5 \\
\hline \multicolumn{5}{|c|}{$x^{5}+184051 x^{4}+8459494809 x^{3}-2471729312 x^{2}+132641 x-1$} \\
\hline 3001 & $\overline{14}$ & 1 & $\overline{14}$ & 11 \\
\hline \multicolumn{5}{|c|}{$\begin{array}{c}x^{5}-4937022 x^{4}-23741798263 x^{3}+2863122794 x^{2} \\
-86142718 x+1 \\
\end{array}$} \\
\hline 3011 & 1 & 1 & 2 & 1 \\
\hline$\overline{3041}$ & $\overline{\mid 1}$ & 1 & $\overline{3}$ & $\overline{1}$ \\
\hline$\overline{3061}$ & $\overline{11}$ & 1 & $\overline{6}$ & $\overline{1}$ \\
\hline$\overline{3091=11.281}$ & $\overline{282,12}$ & $\overline{(1,1)}$ & & $80=16.5$ \\
\hline \multicolumn{5}{|c|}{$x^{5}-430 x^{4}+2867 x^{3}+718 x^{2}+36 x-1$} \\
\hline $3091=11.281$ & 282,12 & $\overline{(2,1)}$ & $\overline{282}$ & 5 \\
\hline \multicolumn{5}{|c|}{$x^{5}+19062309 x^{4}+135905160 x^{3}+27326671 x^{2}+55279 x-1$} \\
\hline $091=11.281$ & 282,12 & $\overline{(3,1)}$ & $282 \pi$ & $\overline{5}$ \\
\hline \multicolumn{5}{|c|}{$\begin{array}{c}x^{5}+7718293463959549075510171 x^{4} \\
+56073876538827712526008456146841450 x^{3} \\
+30923186437566504506062346630 x^{2} \\
-8216463367095091928651 x-1 \\
\end{array}$} \\
\hline
\end{tabular}




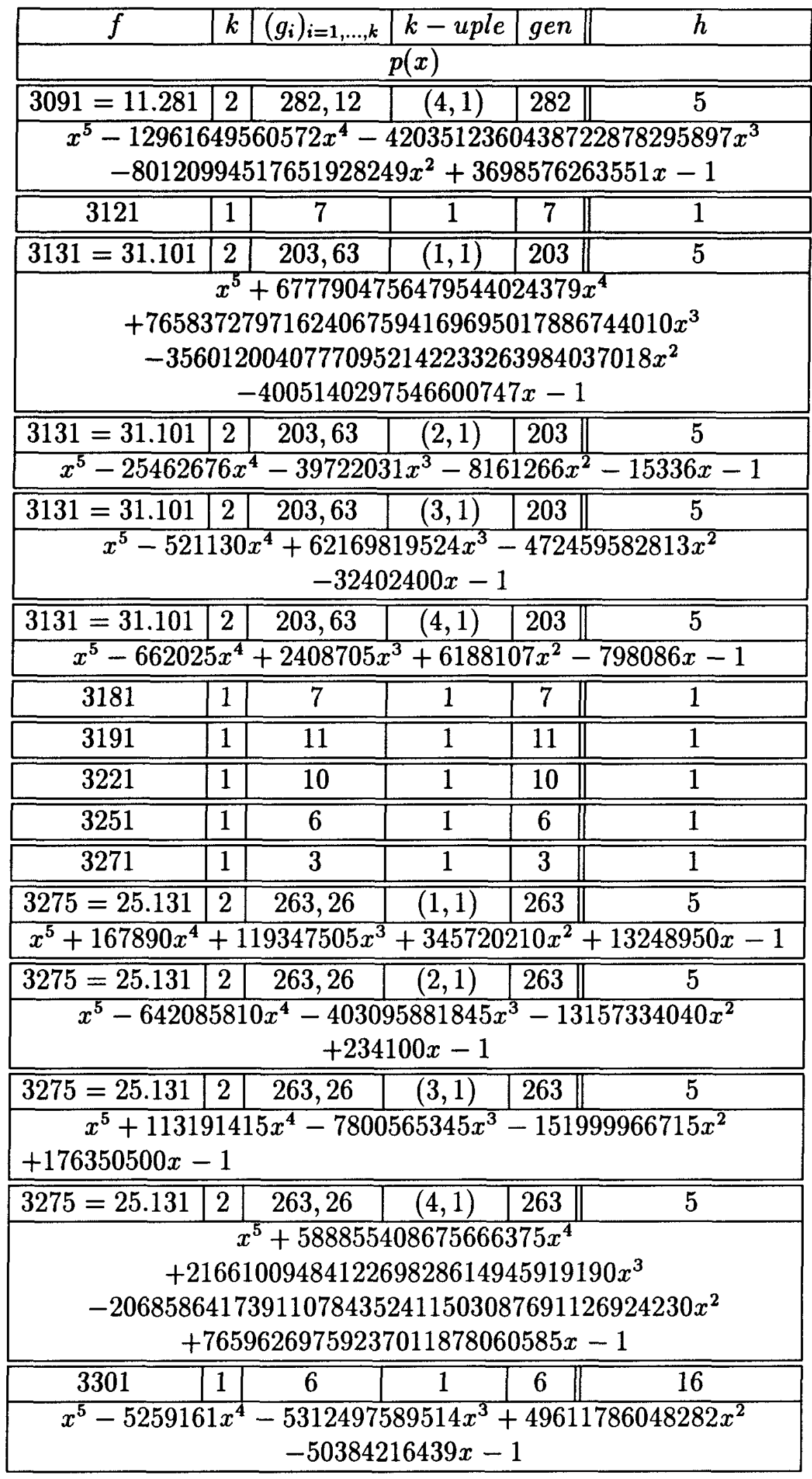




\begin{tabular}{|c|c|c|c|c|c|}
\hline$f$ & $k$ & $\left(g_{i}\right)_{i=1, \ldots, k}$ & $k$-uple & gen & $h$ \\
\hline \multicolumn{6}{|c|}{$p(x)$} \\
\hline (3331 & $\mid$\begin{tabular}{|l|}
1 \\
\end{tabular} & $\overline{\overline{3}}$ & $\overline{1}$ & $\overline{\overline{3}}$ & $\overline{11}$ \\
\hline$\overline{3361}$ & 11 & $\overline{22}$ & $\overline{1}$ & $\overline{22}$ & $\overline{1}$ \\
\hline$\overline{\overline{3371}}$ & 1 & $\overline{\overline{2}}$ & $\overline{1}$ & 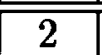 & $\overline{1}$ \\
\hline$\overline{3391}$ & 11 & $\overline{3}$ & $\overline{1}$ & $\overline{3}$ & $\overline{1}$ \\
\hline 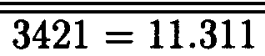 & 2 & $\overline{6623,23}$ & $\overline{\overline{(1,1)}}$ & $\mid 623$ & $\overline{\overline{5}}$ \\
\hline \multicolumn{6}{|c|}{$x^{5}-1735385 x^{4}-645109891242 x^{3}+2726458618 x^{2}-100759 x-1$} \\
\hline $3421=11.311$ & 2 & 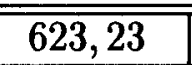 & $\overline{(2,1)}$ & 623 & $\overline{5}$ \\
\hline \multicolumn{6}{|c|}{$\begin{array}{r}x^{5}-91724370552697 x^{4}+3164261357275359165256374 x^{3} \\
-8897320649297355605899193478 x^{2}-198551173271231557303 x-1\end{array}$} \\
\hline $3421=11.311$ & 2 & $\overline{6623,23}$ & $\overline{(3,1)}$ & | & $\overline{55}$ \\
\hline \multicolumn{6}{|c|}{$\begin{array}{c}x^{5}-1988539 x^{4}+314409038017 x^{3}-21338487199 x^{2} \\
-3683700140 x-1\end{array}$} \\
\hline $3421=11.311$ & 2 & 623,23 & $(4,1)$ & 623 & $\overline{5}$ \\
\hline \multicolumn{6}{|c|}{$x^{5}-4359 x^{4}-2848750 x^{3}-407871845 x^{2}+4465563169 x-1$} \\
\hline (3461 & $\begin{array}{ll}1 \\
\end{array}$ & $\overline{\overline{2}}$ & 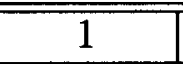 & 2 & 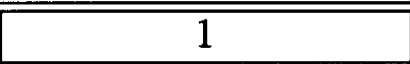 \\
\hline 3491 & 1 & 2 & 1 & 2 & 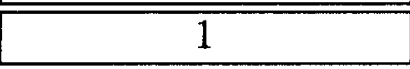 \\
\hline$\overline{3511}$ & 1 & $\overline{7}$ & $\overline{1}$ & $\overline{7}$ & $\overline{11}$ \\
\hline$\overline{3541}$ & 1 & $\overline{7}$ & $\overline{1}$ & 7 & $\overline{1}$ \\
\hline$\overline{\overline{3571}}$ & $\begin{array}{ll}1 \\
1\end{array}$ & $\overline{\overline{2}}$ & $\overline{1}$ & $\overline{2}$ & $\overline{11}$ \\
\hline 3581 & \begin{tabular}{|l|}
1 \\
\end{tabular} & 2 & 1 & 2 & 11 \\
\hline \multicolumn{6}{|c|}{$x^{5}-138489 x^{4}-70760893 x^{3}-1691065513 x^{2}-3279844 x+1$} \\
\hline$\overline{3631}$ & 1 & $\overline{\overline{15}}$ & $\overline{1}$ & $\overline{15}$ & 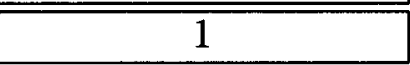 \\
\hline$\overline{3641=11.331}$ & 2 & 332,210 & $\overline{(1,1)}$ & 332|| & 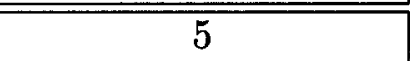 \\
\hline \multicolumn{6}{|c|}{$\begin{array}{c}x^{5}-3858947340209 x^{4}-72782593975658966194258 x^{3} \\
-1310632535234887734200462 x^{2}+1789810499076502220433 x-1\end{array}$} \\
\hline $3641=11.331$ & 2 & 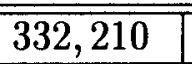 & $\overline{(2,1)}$ & (332 || & $\overline{\overline{5}}$ \\
\hline \multicolumn{6}{|c|}{$\begin{array}{l}x^{5}+4404309872 x^{4}-3497771017078163 x^{3} \\
-14061306331290316 x^{2}+439938808666-1\end{array}$} \\
\hline $3641=11.331$ & 2 & 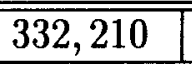 & $\overline{(3,1)}$ & 332 & $\overline{5}$ \\
\hline \multicolumn{6}{|c|}{$x^{5}-62188 x^{4}-91367607 x^{3}+15779209392 x^{2}-774982258 x-1$} \\
\hline$\overline{3641=11.331}$ & \begin{tabular}{|l|}
2 \\
\end{tabular} & 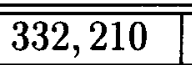 & $\overline{(4,1)}$ & \begin{tabular}{l||}
332 \\
\end{tabular} & $\overline{\overline{5}}$ \\
\hline \multicolumn{6}{|c|}{$\begin{aligned} x^{5}+17083281 x^{4} & +121917985320 x^{3}+5676725335755 x^{2} \\
& +30448293089 x-1\end{aligned}$} \\
\hline$\overline{3671}$ & 1 & $\overline{13}$ & $\overline{1}$ & 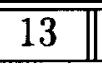 & $\overline{11}$ \\
\hline$\overline{3691}$ & 1 & $\overline{2}$ & $\overline{1}$ & 2 & 1 \\
\hline$\overline{3701}$ & 1 & 2 & 1 & 2 & 1 \\
\hline
\end{tabular}




\begin{tabular}{|c|c|c|c|c|c|}
\hline$f$ & $k$ & $\left(g_{i}\right)_{i=1, \ldots, k}$ & $k-$ uple & gen & $h$ \\
\hline \multicolumn{6}{|c|}{$p(x)$} \\
\hline 3761 & 1 & 3 & 1 & 3 & 1 \\
\hline $3775=25.151$ & 2 & 152,51 & $(1,1)$ & 152 & 5 \\
\hline \multicolumn{6}{|c|}{$x^{5}-175255 x^{4}-8831665515 x^{3}+1876737590 x^{2}+7393755 x-1$} \\
\hline $3775=25.151$ & 2 & 152,51 & $\overline{(2,1)}$ & 152 & 5 \\
\hline \multicolumn{6}{|c|}{$x^{5}-503680 x^{4}-5307815 x^{3}+6523315 x^{2}+772405 x-1$} \\
\hline $3775=25.151$ & 2 & $\overline{152,51}$ & $\overline{(3,1)}$ & 152 & $\overline{5}$ \\
\hline \multicolumn{6}{|c|}{$\begin{array}{c}x^{5}-34252180 x^{4}-1020814465865 x^{3}+1942390094333615 x^{2} \\
+62567252455 x-1\end{array}$} \\
\hline $3775=25.151$ & 2 & 152,51 & $(4,1)$ & 152 & 5 \\
\hline \multicolumn{6}{|c|}{$\begin{array}{c}x^{5}+14120670 x^{4}+14418438083510 x^{3}+1595964415 x^{2} \\
+6080 x-1\end{array}$} \\
\hline 3821 & 1 & 3 & 1 & 3 & 1 \\
\hline 3851 & 1 & 2 & 1 & 2 & 1 \\
\hline$\overline{3881}$ & 1 & $\overline{13}$ & 1 & $\overline{13}$ & $\overline{1}$ \\
\hline 3911 & 1 & 13 & $\overline{1}$ & $\overline{13}$ & $\overline{1}$ \\
\hline$\overline{3931}$ & 1 & $\overline{2}$ & $\overline{1}$ & 2 & $256=16^{2}$ \\
\hline \multicolumn{6}{|c|}{$x^{5}-485 x^{4}+3677 x^{3}+1052 x^{2}+81 x+1$} \\
\hline 4001 & 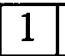 & 3 & 1 & 3 & 1 \\
\hline 4021 & \begin{tabular}{|l|l}
1 & \\
\end{tabular} & 2 & 1 & 2 & 1 \\
\hline 4051 & \begin{tabular}{|l|l}
1 & \\
\end{tabular} & 10 & 1 & 10 & 1 \\
\hline $4061=31.131$ & 2 & 394,187 & $(1,1)$ & 394 & 5 \\
\hline \multicolumn{6}{|c|}{$x^{5}+37594 x^{4}+73453590 x^{3}-186938003 x^{2}-5828662 x-1$} \\
\hline $4061=31.131$ & \begin{tabular}{|l|}
2 \\
\end{tabular} & 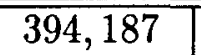 & $\overline{(2,1)}$ & 394 & 5 \\
\hline \multicolumn{6}{|c|}{$x^{5}+118814 x^{4}+118088041 x^{3}+783787193 x^{2}+3856823 x-1$} \\
\hline $4061=31.131$ & 2 & 394,187 & $(3,1)$ & 394 & 5 \\
\hline \multicolumn{6}{|c|}{$\begin{array}{c}x^{5}+775832161137947 x^{4}+60171433322696719512074 x^{3} \\
+505675305573696312053008373958 x^{2}+1380597773114558061525 x \\
-1\end{array}$} \\
\hline $4061=31.131$ & 2 & 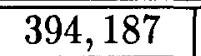 & $\overline{(4,1)}$ & 394 & $\overline{5}$ \\
\hline \multicolumn{6}{|c|}{$\begin{array}{r}x^{5}+193371277610 x^{4}-206175811909204768415 x^{3} \\
-118974505496882754512989 x^{2}+1065915795097237 x\end{array}$} \\
\hline 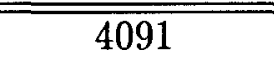 & 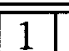 & 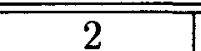 & 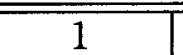 & 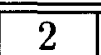 & 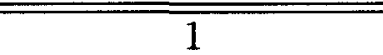 \\
\hline$\overline{4111}$ & \begin{tabular}{l|l}
1 &
\end{tabular} & $\overline{12}$ & $\overline{1}$ & 12 & $\overline{c 1}$ \\
\hline $4141=41.101$ & 2 & 304,42 & $(1,1)$ & 304 & 5 \\
\hline \multicolumn{6}{|c|}{$x^{5}-4313806 x^{4}+2141182335 X^{3}+7443147015 x^{2}+45859 x-1$} \\
\hline $4141=41.101$ & $2 \mid$ & 304,42 & $\overline{(2,1)}$ & 304 & 5 \\
\hline
\end{tabular}




\begin{tabular}{|c|c|c|c|c|}
\hline$f$ & \begin{tabular}{l|l|}
$k$ & $\left(g_{i}\right)_{i=1, \ldots, k}$ \\
\end{tabular} & $k-$ uple & gen & $\bar{h}$ \\
\hline \multicolumn{5}{|c|}{$p(x)$} \\
\hline \begin{tabular}{|l|l|}
$4141=41.101$ & 2 \\
\end{tabular} & \begin{tabular}{|l|l|}
2 & 304,42 \\
\end{tabular} & $(3,1)$ & 304 & 5 \\
\hline \multicolumn{5}{|c|}{$\begin{array}{c}x^{5}-1719211787292049144672037345 x^{4} \\
-162019059592015531711552791090314242 x^{3} \\
+5256956489315048499662841826 x^{2}+197587322514518529 x-1\end{array}$} \\
\hline $\begin{array}{l}4141=41.101 \\
2\end{array}$ & $\overline{304,42}$ & $\overline{(4,1)}$ & 304 & $\overline{5}$ \\
\hline \multicolumn{5}{|c|}{$\begin{aligned} x^{5}-32797836 x^{4}+ & 1179408856117 x^{3}-4631678484224 x^{2} \\
& -109876986 x-1\end{aligned}$} \\
\hline 4201 & $\overline{11}$ & $\overline{1}$ & $\overline{11}$ & $\overline{11}$ \\
\hline \multicolumn{5}{|c|}{$\begin{array}{c}x^{5}+425715305661 x^{4}-1734269244369415 x^{3} \\
-6477825888243331 x^{2}+144276708 x+1 \\
\end{array}$} \\
\hline 4211 & 6 & 1 & 6 & 1 \\
\hline 4231 & 3 & 1 & 3 & 1 \\
\hline 4241 & 3 & 1 & 3 & 1 \\
\hline 4261 & 2 & 1 & 2 & 1 \\
\hline 4271 & 7 & 1 & 7 & 1 \\
\hline \begin{tabular}{l|l}
$4331=61.71$ & 2 \\
\end{tabular} & 214,62 & $(1,1)$ & 214 & 5 \\
\hline \multicolumn{5}{|c|}{$\begin{array}{c}x^{5}+106344311 x^{4}-400429188572646 x^{3}+71424294577655 x^{2} \\
-1104024741 x-1\end{array}$} \\
\hline $4331=61.71$ & 214,62 & $\overline{(2,1)}$ & 214 & 5 \\
\hline \multicolumn{5}{|c|}{$\begin{array}{c}x^{5}-1274619488062 x^{4}+48177835229832254759 x^{3} \\
-605365915545087 x^{2}-373272435 x-1\end{array}$} \\
\hline \begin{tabular}{l|l|}
$4331=61.71$ & 2 \\
\end{tabular} & 214,62 & $(3,1)$ & 214 & 5 \\
\hline \multicolumn{5}{|c|}{$\begin{array}{c}x^{5}+50472504244002406036525385144183 x^{4} \\
-27345554503996562895120152876624974437434 x^{3} \\
-298984016169878483233162422646 x^{2}+717132397414207033 x-1 \\
\end{array}$} \\
\hline \begin{tabular}{|l|l|}
$4331=61.71$ & 2 \\
\end{tabular} & \begin{tabular}{|l|l|}
214,62 \\
\end{tabular} & $\overline{(4,1)}$ & 214|| & $\overline{5}$ \\
\hline \multicolumn{5}{|c|}{$x^{5}+20716110 x^{4}+7555373 x^{3}+435938 x^{2}+3462 x-1$} \\
\hline 4391 & 14 & 1 & 14 & 1 \\
\hline \begin{tabular}{|l|l|}
$4411=11.401$ & 2 \\
\end{tabular} & 402,12 & $(1,1)$ & 402 & $55=5.11$ \\
\hline \multicolumn{5}{|c|}{$x^{5}+3222 x^{4}+47637 x^{3}-6806 x^{2}+256 x-1$} \\
\hline \begin{tabular}{|l|l|}
$4411=11.401$ & 2 \\
\end{tabular} & 402,12 & $(2,1)$ & 402 & 5 \\
\hline \multicolumn{5}{|c|}{$\begin{array}{c}x^{5}+1572662560293566962915 x^{4} \\
+33192784549384155847800164302914 x^{3} \\
+8824145646175423406926512449502 x^{2}-3442453080541967235 x-1 \\
\end{array}$} \\
\hline \begin{tabular}{|l|l|}
$4411=11.401$ & 2 \\
\end{tabular} & 402,12 & $\overline{(3,1)}$ & 402 & 5 \\
\hline$x-40$ & $\begin{array}{r}1 x^{4}+219 \\
-4 \\
\end{array}$ & $\begin{array}{l}692035 x \\
774732 x \\
\end{array}$ & & $38607 x^{2}$ \\
\hline
\end{tabular}




\begin{tabular}{|c|c|c|c|c|c|}
\hline$f$ & $k$ & $\left(g_{i}\right)_{i=1, \ldots, k}$ & $k-u p l e$ & gen & $h$ \\
\hline \multicolumn{6}{|c|}{$p(x)$} \\
\hline $4411=11.401$ & 2 & 402,12 & $(4,1)$ & 402 & 5 \\
\hline \multicolumn{6}{|c|}{$\begin{array}{c}x^{5}+8073252988025733 x^{4}+33604409015418525953542952249 x^{3} \\
-247475053101459476207 x^{2}+97803461100 x-1 \\
\end{array}$} \\
\hline 4421 & \begin{tabular}{|l|l|}
1 & \\
\end{tabular} & 3 & 1 & 3 & 1 \\
\hline 4441 & 1 & 21 & 1 & 21 & 1 \\
\hline$\overline{4451}$ & \begin{tabular}{l|l}
1 \\
\end{tabular} & 2 & 1 & 2 & 1 \\
\hline 4481 & \begin{tabular}{l|l}
1 \\
\end{tabular} & 3 & 1 & $\overline{3}$ & 1 \\
\hline $4525=25.181$ & 2 & 363,76 & $(1,1)$ & \begin{tabular}{|l||}
363 \\
\end{tabular} & 5 \\
\hline \multicolumn{6}{|c|}{$\begin{array}{r}x^{5}+2633239815 x^{4}-3010822748902045 x^{3} \\
+35689177402220860 x^{2}-429947937275 x-1 \\
\end{array}$} \\
\hline $4525=25.181$ & 2 & 363,76 & $(2,1)$ & \begin{tabular}{|l||}
363 \\
\end{tabular} & 5 \\
\hline \multicolumn{6}{|c|}{$\begin{array}{c}x^{5}+2370485664431365 x^{4}-557652413897763034345 x^{3} \\
-216428760602280209365 x^{2}-240825032850 x-1\end{array}$} \\
\hline $4525=25.181$ & 2 & 363,76 & $(3,1)$ & 363 & 5 \\
\hline \multicolumn{6}{|c|}{$\begin{array}{r}x^{5}-55552428605774226585 x^{4}+90266407964264322412630 x^{3} \\
+8724649755663510628150810 x^{2}-141991784395692286434775 x-1\end{array}$} \\
\hline $4525=25.181$ & $\overline{\overline{2}}$ & $\overline{c 363,76}$ & $(4,1)$ & 363 & $\overline{5}$ \\
\hline \multicolumn{6}{|c|}{$\begin{array}{c}x^{5}+159198283895840 x^{4}-18297722330541866521145 x^{3} \\
+57823644094271660 x^{2}+27970628050 x-1\end{array}$} \\
\hline 4561 & 1 & 11 & 1 & 11 & 1 \\
\hline 4591 & 1 & 11 & 1 & 11 & 1 \\
\hline 4621 & 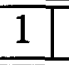 & 2 & 1 & 2 & 1 \\
\hline $4631=11.421$ & 2 & 843,23 & $(1,1)$ & 843 & 5 \\
\hline \multicolumn{6}{|c|}{$x^{5}-38090814 x^{4}-714468824 x^{3}-260250393 x^{2}+168532 x-1$} \\
\hline $4631=11.421$ & 2 & 843,23 & $(2,1)$ & 843 & 5 \\
\hline \multicolumn{6}{|c|}{$\begin{array}{l}x^{5}-17612209761592933 x^{4}-704104489521260578095194 x^{3} \\
+42702291263854073164493 x^{2}+3813853065863897233 x-1 \\
\end{array}$} \\
\hline $4631=11.421$ & 2 & 843,23 & $(3,1)$ & 843 & 5 \\
\hline \multicolumn{6}{|c|}{$\begin{aligned} x^{5}+8052470 x^{4}+ & 279486376639 x^{3}+4707542624679 x^{2} \\
& +1124209483 x-1\end{aligned}$} \\
\hline $4631=11.421$ & 2 & 843,23 & $\overline{(4,1)}$ & 843 & 5 \\
\hline \multicolumn{6}{|c|}{$\begin{array}{c}x^{5}+2553036884657736195979719 x^{4} \\
-31426859901036219672370754017354 x^{3}\end{array}$} \\
\hline & \multicolumn{3}{|c|}{$-55454004119737686916504809285062 x^{2}+343233343688524873 x-1$} & & \\
\hline & 1 & 3 & 1 & 3 & 1 \\
\hline
\end{tabular}




\begin{tabular}{|c|c|c|c|c|}
\hline$f$ & \begin{tabular}{l|l}
$k$ & $\left(g_{i}\right)_{i=1}$ \\
\end{tabular} & $k$-uple & gen & $h$ \\
\hline \multicolumn{5}{|c|}{$p(x)$} \\
\hline \begin{tabular}{l|l|l|}
$681=31.151$ & 2 \\
\end{tabular} & $\overline{303,63}$ & $\overline{(1,1)}$ & 303 & 5 \\
\hline \multicolumn{5}{|c|}{$x^{5}-292665522 x^{4}-108872728914 x^{3}-766420569 x^{2}-288466 x-$} \\
\hline \begin{tabular}{|l|l|}
$4681=31.151$ & 2
\end{tabular} & 303,63 & $\overline{(2,1)}$ & (303 & $\overline{5}$ \\
\hline \multicolumn{5}{|c|}{$x^{5}-5132735264 x^{4}-15899956741 x^{3}-86304036 x^{2}+3727832 x-$} \\
\hline \begin{tabular}{|l|l|}
$4681=31.151$ & 2
\end{tabular} & $\overline{303,63}$ & $\overline{(3,1)}$ & 303 & $\overline{55}$ \\
\hline \multicolumn{5}{|c|}{$\begin{array}{r}x^{5}-11249378563424 x^{4}+35023726742569663461895 x^{3} \\
+41363351469726779522465 x^{2}+6592707026958273251 x-1\end{array}$} \\
\hline \begin{tabular}{l|l|l}
$4681=31.151$ & 2
\end{tabular} & 2303,63 & $(4,1)$ & \begin{tabular}{|c|}
303 \\
\end{tabular} & 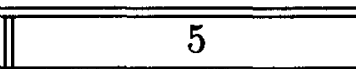 \\
\hline \multicolumn{5}{|c|}{$\begin{array}{c}x^{5}-5079177838719 x^{4}-256175741853988281 x^{3} \\
-5866824685012567991 x^{2}+7085594008 x-1\end{array}$} \\
\hline 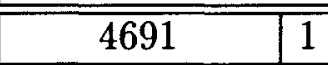 & $\overline{22}$ & 1 & 2 & 1 \\
\hline$\overline{4721}$ & $\overline{6}$ & $\overline{11}$ & $\overline{6}$ & 1 \\
\hline \begin{tabular}{l|l}
$741=11.431$ & 2 \\
\end{tabular} & \begin{tabular}{|l|l|}
2 & 1294,34 \\
\end{tabular} & $\bar{~} \overline{(1,1)}$ & 1294 & \\
\hline \multicolumn{5}{|c|}{$\begin{array}{c}x^{5}-5822249 x^{4}-1853877960361 x^{3}+18119289908 x^{2} \\
+169101 x-1\end{array}$} \\
\hline \begin{tabular}{|l|l|}
$741=11.431$ & 2 \\
\end{tabular} & \begin{tabular}{l|l}
2 & 1294,34
\end{tabular} & $\overline{\overline{(2,1)}}$ & $\mid$ & $\overline{5}$ \\
\hline \multicolumn{5}{|c|}{$\begin{array}{c}x^{5}+8879592 x^{4}-2919866959 x^{3}+60796306939 x^{2} \\
+1240395737 x-1\end{array}$} \\
\hline \begin{tabular}{|l|l|}
$4741=11.431$ & 2
\end{tabular} & \begin{tabular}{|l|l}
1294,34 \\
\end{tabular} & $\overline{(3,1)}$ & 1294 & $\overline{5}$ \\
\hline \multicolumn{5}{|c|}{$x^{5}-351135 x^{4}+17850648422 x^{3}+88359087085 x^{2}-9768035 x-$} \\
\hline \begin{tabular}{l|l}
$4741=11.431$ & 2 \\
\end{tabular} & \begin{tabular}{|l|l|}
2 & 1294,34 \\
\end{tabular} & $\overline{(4,1)}$ & 1294 & $\overline{5}$ \\
\hline \multicolumn{5}{|c|}{$\begin{array}{c}x^{5}+1177373399657451 x^{4}+28798705861192005 x^{3} \\
+769821527265565 x^{2}-1191566 x-1\end{array}$} \\
\hline 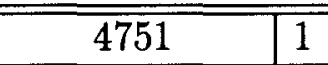 & $\overline{19}$ & 1 & $\overline{19}$ & 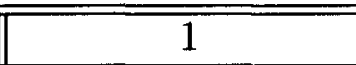 \\
\hline \begin{tabular}{l|l}
$775=25.191$ & 2 \\
\end{tabular} & \begin{tabular}{l|l}
192,76 \\
\end{tabular} & $\overline{(1,1)}$ & 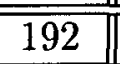 & 5 \\
\hline \multicolumn{5}{|c|}{$\begin{aligned} x^{5}-52854826445 x^{4}+ & 596718969245 x^{3}+14093747100475 x^{2} \\
& +7312440 x-1\end{aligned}$} \\
\hline \begin{tabular}{l|l|}
$75=25.191$ & 2 \\
\end{tabular} & $\overline{192,76}$ & $\overline{(2,1)}$ & 192 & $\overline{5}$ \\
\hline \multicolumn{5}{|c|}{$\begin{array}{c}x^{5}+542846324549055 x^{4}+92316015366832294338270 x^{3} \\
-7022956132057446856604489365950 x^{2} \\
+1804540134551360306465 x-1\end{array}$} \\
\hline \begin{tabular}{l|l|l}
$=25.191$ & 2 \\
\end{tabular} & 192,76 & $\overline{(3,1)}$ & $\overline{192}$ & $\overline{55}$ \\
\hline \multicolumn{5}{|c|}{$\begin{array}{c}x^{5}-3388345 x^{4}-26067701955 x^{3}-80366905750 x^{2} \\
+6029509015 x-1\end{array}$} \\
\hline \begin{tabular}{l|l}
$75=25.191$ & 2 \\
\end{tabular} & 192,76 & $\overline{(4,1)}$ & 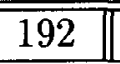 & $\overline{5}$ \\
\hline I & $\begin{array}{r}5 x^{4}+536 \\
-7150\end{array}$ & 974804 & $3-2$ & \\
\hline
\end{tabular}




\begin{tabular}{|c|c|c|c|c|c|}
\hline$f$ & $k$ & $\left(g_{i}\right)_{i=1, \ldots, k}$ & $k-$ uple & gen & $h$ \\
\hline \multicolumn{6}{|c|}{$p(x)$} \\
\hline$\overline{4801}$ & \begin{tabular}{|l|l|}
1 \\
\end{tabular} & $\overline{7}$ & $\overline{\overline{1}}$ & $\overline{\overline{7}}$ & 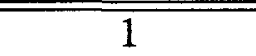 \\
\hline$\overline{4831}$ & 1 & $\overline{\overline{3}}$ & $\overline{1}$ & $\overline{3}$ & $\overline{1}$ \\
\hline 4861 & \begin{tabular}{l|l}
1 \\
\end{tabular} & $\overline{11}$ & $\overline{11}$ & 11 & $\overline{11}$ \\
\hline 4871 & \begin{tabular}{|l|l|}
1 & \\
\end{tabular} & 11 & 1 & 11 & $\overline{1}$ \\
\hline$\overline{4931}$ & 1 & $\overline{\overline{6}}$ & $\overline{11}$ & 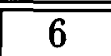 & 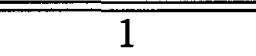 \\
\hline 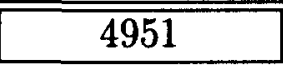 & 1 & $\overline{6}$ & 1 & 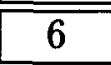 & 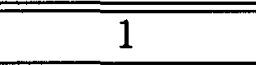 \\
\hline 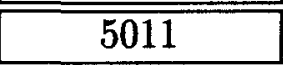 & 1 & $\overline{22}$ & $\overline{1}$ & 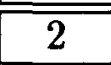 & 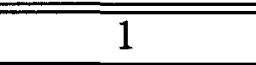 \\
\hline 5021 & $\overline{\mid 1}$ & $\overline{\overline{3}}$ & 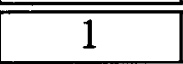 & $\overline{3}$ & $\overline{11}$ \\
\hline$\overline{\overline{5051}}$ & 11 & 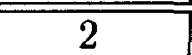 & $\overline{\overline{1}}$ & $\overline{22}$ & 1451 \\
\hline \multicolumn{6}{|c|}{$x^{5}+49 x^{4}-1060 x^{3}+4765 x^{2}+619 x+1$} \\
\hline $5071=11.461$ & $2 \mid$ & 1845,12 & $(1,1)$ & 1845 & $\overline{5}$ \\
\hline \multicolumn{6}{|c|}{$\begin{array}{c}x^{5}+5098220 x^{4}-134844698517 x^{3}-88227387577 x^{2} \\
+920067 x-1\end{array}$} \\
\hline $5071=11.461$ & 2 & 1845,12 & $(2,1)$ & 1845 & 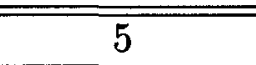 \\
\hline \multicolumn{6}{|c|}{$\begin{array}{c}x^{5}-17381523 x^{4}+1979529106477 x^{3}-7159398211667910 x^{2} \\
-12008080145 x-1\end{array}$} \\
\hline $5071=11.461$ & $2 \mid$ & 1845,12 & $(3,1)$ & 1845 & 5 \\
\hline \multicolumn{6}{|c|}{$\begin{array}{c}x^{5}+1399221325863055740348 x^{4} \\
+4070761609772128966090343309 x^{3} \\
+603897339451442018840683 x^{2}-753655116165 x-1\end{array}$} \\
\hline $5071=11.461$ & 2 & 1845,12 & $\overline{(4,1)}$ & 1845 & $80=16.5$ \\
\hline \multicolumn{6}{|c|}{$\begin{aligned} x^{5}-1648993 x^{4}- & 41809609634 x^{3}-224514602314078 x^{2} \\
& +24098839649 x-1\end{aligned}$} \\
\hline$\overline{5081}$ & \begin{tabular}{l|l}
1 \\
\end{tabular} & $\overline{3}$ & $\overline{1}$ & 3 & 1 \\
\hline$\overline{\overline{5101}}$ & $\overline{1}$ & $\overline{\overline{6}}$ & $\overline{\overline{1}}$ & $\overline{6}$ & $\overline{11}$ \\
\hline$\overline{5171}$ & 1 & $\overline{2}$ & $\overline{1}$ & 2 & $\overline{1}$ \\
\hline$\overline{5231}$ & 1 & $\overline{7}$ & $\overline{1}$ & $\overline{7}$ & $\overline{1}$ \\
\hline$\overline{5261}$ & $\overline{1}$ & 2 & $\overline{1}$ & $\overline{2}$ & $\overline{1}$ \\
\hline $5275=25.211$ & \begin{tabular}{l|l}
2 & \\
\end{tabular} & $\overline{212,376}$ & $\overline{(1,1)}$ & 212 & $\overline{5}$ \\
\hline \multicolumn{6}{|c|}{$\begin{array}{c}x^{5}+1060708449613385 x^{4}-106278551865893536750 x^{3} \\
-91830185782272669245 x^{2}+2352306348795 x-1\end{array}$} \\
\hline$\overline{5275=25.211}$ & \begin{tabular}{l|l}
2 & \\
\end{tabular} & $\overline{\overline{212,376}}$ & $\overline{(2,1)}$ & 212 & $\overline{5}$ \\
\hline \multicolumn{6}{|c|}{$\begin{array}{c}x^{5}+7015090582115805185 x^{4}-3154016357221041833649950 x^{3} \\
+60003254608283770438655 x^{2}-203959319955155 x-1\end{array}$} \\
\hline$\overline{5275=25.211}$ & $\overline{2}$ & $\overline{212,376}$ & $\overline{(3,1)}$ & 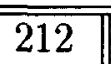 & $\overline{\overline{5}}$ \\
\hline $\begin{array}{r}x^{5}-142519371 \\
+7698991515 \\
\end{array}$ & & $4594265 x^{4}$ & $\begin{array}{l}-2856361 \\
330 x^{2}-46\end{array}$ & 20916 & $\begin{array}{l}56276615850 x^{3} \\
639255 x-1\end{array}$ \\
\hline
\end{tabular}




\begin{tabular}{|c|c|c|c|c|c|}
\hline$f$ & $k$ & $\left(g_{i}\right)_{i=1, \ldots, k}$ & $k$-uple & gen & $n$ \\
\hline \multicolumn{6}{|c|}{$p(x)$} \\
\hline $75=25.211$ & 2 & 212,376 & $(4,1)$ & \begin{tabular}{|l||}
212 \\
\end{tabular} & 5 \\
\hline \multicolumn{6}{|c|}{$\begin{array}{c}x^{5}+389410 x^{4}+19900720325 x^{3}-17451359170 x^{2} \\
-2125962930 x-1\end{array}$} \\
\hline 5281 & 1 & $\overline{7}$ & 1 & 7 & 1 \\
\hline 5351 & & 11 & & 11 & \\
\hline $371=41.131$ & 2 & 263,83 & $(1,1)$ & 263 & \\
\hline \multicolumn{6}{|c|}{$\begin{array}{r}x^{5}+3228753790565448 x^{4}-39282606720720662096486 x^{3} \\
+1217159094079896129295063 x^{2}+254298893847950 x-1 \\
\end{array}$} \\
\hline $3371=41.131$ & 2 & $\overline{263,83}$ & $\overline{(2,1)}$ & 263 & $\overline{5}$ \\
\hline \multicolumn{6}{|c|}{$\begin{array}{r}x^{5}+80642360538445051 x^{4}+1101828538644243899562 x^{3} \\
+6784404265417391746662 x^{2}+7654873619767426741 x-1 \\
\end{array}$} \\
\hline $5371=41.131$ & 2 & 263,83 & $(3,1)$ & 263 & 5 \\
\hline$x^{5}-19577154$ & & -1085036 & $627 x^{3}-2$ & 055184 & $4 x^{2}+3278 x-1$ \\
\hline $5371=41.131$ & 2 & 263,83 & $(4,1)$ & 263 & 5 \\
\hline \multicolumn{6}{|c|}{$\begin{array}{c}x^{5}+30705338979 x^{4}-142383702009134 x^{3}-2274510906219 x^{2} \\
-1244699117 x-1\end{array}$} \\
\hline 5381 & 1 & 3 & 1 & 3 & 1 \\
\hline $401=11.491$ & 4 & 492,23 & $(1,1)$ & 492 & 5 \\
\hline \multicolumn{6}{|c|}{$\begin{array}{c}x^{5}-5554616 x^{4}+1826992385236 x^{3}+1266225587627201 x^{2} \\
-307327270 x-1 \\
\end{array}$} \\
\hline $101=11.491$ & \begin{tabular}{|l|}
2 \\
\end{tabular} & 492,23 & $(2,1)$ & 492 & 5 \\
\hline \multicolumn{6}{|c|}{$\begin{array}{c}x^{5}-42105123589 x^{4}+171663465200 x^{3}+487847489605 x^{2} \\
-555454611 x-1 \\
\end{array}$} \\
\hline $401=11.491$ & \begin{tabular}{|l|l|}
2 & \\
\end{tabular} & 492,23 & $(3,1)$ & 492 & 5 \\
\hline \multicolumn{6}{|c|}{$\begin{array}{r}x^{5}-18993877321 x^{4}-280740316501685242 x^{3} \\
+9656547975469337418 x^{2}+683659556021353927161 x-1 \\
\end{array}$} \\
\hline $401=11.491$ & \begin{tabular}{|l|l|}
2 & \\
\end{tabular} & 492,23 & $(4,1)$ & \begin{tabular}{l||}
492 \\
\end{tabular} & 5 \\
\hline \multicolumn{6}{|c|}{$\begin{array}{c}x^{5}-1063221644 x^{4}-84963569926 x^{3}-1007271493 x^{2} \\
-64380 x-1 \\
\end{array}$} \\
\hline 5431 & \begin{tabular}{l|l}
1 & \\
\end{tabular} & 3 & 1 & $\overline{3}$ & $\overline{11}$ \\
\hline 5501 & 1 & 2 & 1 & 2 & 11 \\
\hline \multicolumn{6}{|c|}{$\begin{array}{c}x^{5}-459734712 x^{4}-815797600954 x^{3}+46227492317 x^{2} \\
+434009898 x+1 \\
\end{array}$} \\
\hline 5521 & 1 & 11 & 1 & 11 & $\overline{1}$ \\
\hline 5531 & 1 & 10 & 1 & 10 & 1 \\
\hline 5581 & & & & 6 & \\
\hline
\end{tabular}




\begin{tabular}{|c|c|c|c|c|c|}
\hline$f$ & $k$ & $\left(g_{i}\right)_{i=1, \ldots, k}$ & $k-$ uple & gen & $h$ \\
\hline \multicolumn{6}{|c|}{$p(x)$} \\
\hline 5591 & 1 & 11 & 1 & 11 & 1 \\
\hline $5611=31.181$ & 2 & $\overline{363,63}$ & $\overline{(1,1)}$ & 363 & $\overline{5}$ \\
\hline \multicolumn{6}{|c|}{$\begin{array}{c}x^{5}-109327058882557 x^{4}-26302099932973559847727646 x^{3} \\
-105308802186637677619878778382658 x^{2} \\
-6475184254095075837282083 x-1 \\
\end{array}$} \\
\hline $5611=31.181$ & 2 & $\overline{363,63}$ & $\overline{(2,1)}$ & $\overline{363}$ & $\overline{5}$ \\
\hline \multicolumn{6}{|c|}{$x^{5}+426865 x^{4}+13082516253 x^{3}-109387695 x^{2}-48014 x-1$} \\
\hline $5611=31.181$ & 2 & 363,63 & $(3,1)$ & 363 & 5 \\
\hline \multicolumn{6}{|c|}{$x^{5}+17262 x^{4}+40796010 x^{3}-5475882759 x^{2}+498062900 x-1$} \\
\hline $5611=31.181$ & 2 & 363,63 & $\overline{(4,1)}$ & 363 & $55=5.11$ \\
\hline \multicolumn{6}{|c|}{$x^{5}-1970086 x^{4}+103275208 x^{3}+258319859 x^{2}-132644 x-1$} \\
\hline 5641 & 1 & 14 & 1 & $\overline{14}$ & 1 \\
\hline 5651 & 1 & 2 & 1 & 2 & 1 \\
\hline 5701 & 1 & 2 & 1 & 2 & 1 \\
\hline 5711 & 1 & 19 & 1 & 19 & 1 \\
\hline $5731=11.521$ & 2 & 1564,23 & $(1,1)$ & 1564 & 5 \\
\hline \multicolumn{6}{|c|}{$\begin{array}{c}x^{5}+1911604987144749849551487567 x^{4} \\
-20219265718209844135497207042066564578190706 x^{3} \\
+487813638649386336760177880334263733490 x^{2} \\
+61346391571610997171761 x-1\end{array}$} \\
\hline $5731=11.521$ & 2 & $\overline{1564,23}$ & $\overline{(2,1)}$ & 1564 & $\overline{5}$ \\
\hline \multicolumn{6}{|c|}{$\begin{array}{c}x^{5}-633159101281 x^{4}+81812286584011818788 x^{3} \\
+29542800067616763 x^{2}-283890652407 x-1\end{array}$} \\
\hline $5731=11.521$ & 2 & 1564,23 & $(3,1)$ & 1564 & 5 \\
\hline \multicolumn{6}{|c|}{$x^{5}+4621295 x^{4}-5478451773 x^{3}+2796850643 x^{2}+153427726 x-1$} \\
\hline $5731=11.521$ & 2 & $\overline{1564,23}$ & $\overline{(4,1)}$ & 1564 & $\overline{5}$ \\
\hline \multicolumn{6}{|c|}{$\begin{array}{c}x^{5}-5467977159589445647 x^{4} \\
-1216455385340959940802657617 x^{3}+38609075999327685620531 x^{2} \\
-1414142717888 x-1 \\
\end{array}$} \\
\hline 5741 & 1 & 2 & 1 & 2 & 1 \\
\hline 5791 & $1 \mid$ & 6 & 1 & 6 & 1 \\
\hline 5801 & 1 & 3 & 1 & 3 & 1 \\
\hline 5821 & \begin{tabular}{l|l}
1 \\
\end{tabular} & 6 & 1 & 6 & 1 \\
\hline 5851 & 11 & 2 & 1 & 2 & 1 \\
\hline 5861 & \begin{tabular}{l|l|}
1 \\
\end{tabular} & 3 & 1 & 3 & 1 \\
\hline 5881 & \begin{tabular}{l|}
1 \\
\end{tabular} & 31 & 1 & 31 & $\overline{1}$ \\
\hline
\end{tabular}




\begin{tabular}{|c|c|c|c|c|c|}
\hline $\bar{f}$ & $k$ & $\left(g_{i}\right)_{i=1, \ldots, k}$ & $k-u p l e$ & gen & $h$ \\
\hline \multicolumn{6}{|c|}{$p(x)$} \\
\hline $5921=31.191$ & 2 & 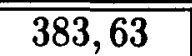 & $\overline{(1,1)}$ & $\overline{383}$ & $\overline{\overline{25}}$ \\
\hline \multicolumn{6}{|c|}{$\begin{array}{c}x^{5}+4521147658 x^{4}+19221476199 x^{3}-1524302157 x^{2} \\
-341925 x-1\end{array}$} \\
\hline $5921=31.191$ & 2 & 383,63 & $\overline{(2,1)}$ & 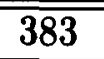 & 25 \\
\hline \multicolumn{6}{|c|}{$\begin{array}{l}x^{5}-9980950407 x^{4}-137624905461643681 x^{3} \\
-111955750364981117 x^{2}-1110203770 x-1\end{array}$} \\
\hline$\overline{5921=31.191}$ & 2 & $\overline{383,63}$ & $\overline{(3,1)}$ & 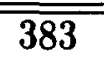 & $\overline{\overline{25}}$ \\
\hline \multicolumn{6}{|c|}{$\begin{array}{c}x^{5}+107741952500 x^{4}+37857012522 x^{3}-40750169269 x^{2} \\
+142395622 x-1\end{array}$} \\
\hline $5921=31.191$ & 2 & 383,63 & $\overline{(4,1)}$ & 383 & 25 \\
\hline \multicolumn{6}{|c|}{$\begin{array}{c}x^{5}+13431359661124868630519605042119 x^{4} \\
-40045233481472048212858011181636178820576317578 x^{3} \\
+284819964574405216794978379026467962335418 x^{2} \\
-65151846558067165913079 x-1\end{array}$} \\
\hline $5951=11.541$ & 2 & 1624,67 & $\overline{(1,1)}$ & 1624 & $\overline{25}$ \\
\hline \multicolumn{6}{|c|}{$\begin{array}{l}x^{5}+9784454303769781526491 x^{4} \\
21284675773976150652184624944042 x^{3} \\
66743471278350662 x^{2}+403427046504821 x-1\end{array}$} \\
\hline $5951=11.541$ & 2 & 1624,67 & $\overline{(2,1)}$ & 1624 & 25 \\
\hline \multicolumn{6}{|c|}{$\begin{array}{c}x^{5}-29162610 x^{4}-4753970406 x^{3}-4280773415 x^{2} \\
-260384918 x-1\end{array}$} \\
\hline $5951=11.541$ & $\overline{2}$ & $16 \overline{1624,67}$ & $\overline{(3,1)}$ & 1624 & $\overline{25}$ \\
\hline \multicolumn{6}{|c|}{$\begin{array}{r}x^{5}-21228970131604 x^{4}-4451873800125950861234339 x^{3} \\
+63383209486147394781336965 x^{2}-82743938648846128403 x-1 \\
\end{array}$} \\
\hline $5951=11.541$ & $\overline{22}$ & 1624,67 & $\overline{(4,1)}$ & 1624 & $\overline{25}$ \\
\hline \multicolumn{6}{|c|}{$\begin{array}{l}x^{5}-228696533993 x^{4}-456273339469671 x^{3} \\
+14132089452640 x^{2}+191735591441 x-1\end{array}$} \\
\hline$\overline{5981}$ & $\overline{1}$ & 3 & 1 & $\overline{3}$ & 1 \\
\hline 6011 & $\overline{1}$ & 2 & $\overline{1}$ & $\overline{2}$ & $\overline{1}$ \\
\hline $6025=25.241$ & \begin{tabular}{l|l}
2 &
\end{tabular} & 242,51 & $\overline{(1,1)}$ & $\overline{242}$ & $\overline{5}$ \\
\hline \multicolumn{6}{|c|}{$\begin{array}{l}x^{5}+814818665480 x^{4}+82904685991050072995 x^{3} \\
-30747934750869964250 x^{2}+25604097690 x-1\end{array}$} \\
\hline $25=25.241$ & \begin{tabular}{l|l}
2 &
\end{tabular} & 242,51 & $(2,1)$ & 242 & $\overline{5}$ \\
\hline \multicolumn{6}{|c|}{$\begin{array}{c}x^{5}-292045 x^{4}+6617635645 x^{3}-26033836271400 x^{2} \\
-2185449635 x-1\end{array}$} \\
\hline $025=25.241$ & $\overline{\overline{2}}$ & $\overline{242,51}$ & $\overline{(3,1)}$ & $\overline{242}$ & $\overline{55}$ \\
\hline \multicolumn{6}{|c|}{$\begin{array}{l}x^{5}+8903465005 x^{4}-1201987574124769080 x^{3} \\
15985769909459970545625 x^{2}-261870673685 x-1\end{array}$} \\
\hline
\end{tabular}




\begin{tabular}{|c|c|c|c|c|c|}
\hline$f$ & & $\left(g_{i}\right)_{i=1, \ldots, k}$ & $k$-uple & gen & $\bar{h}$ \\
\hline \multicolumn{6}{|c|}{$p(x)$} \\
\hline $6025=25.241$ & 2 & $\overline{242,51}$ & $\overline{(4,1)}$ & $\mid 242$ & $\overline{5}$ \\
\hline \multicolumn{6}{|c|}{$\begin{array}{c}x^{5}+100862867682330 x^{4}-10189363927276878446455 x^{3} \\
+1408094450459706675175 x^{2}-4727852904285 x-1\end{array}$} \\
\hline 6091 & \begin{tabular}{|l|}
1 \\
1
\end{tabular} & 7 & 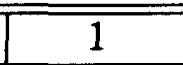 & 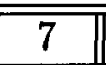 & 1 \\
\hline$\overline{6101}$ & 1 & $\overline{2}$ & $\overline{11}$ & $\overline{2}$ & $\overline{\overline{1}}$ \\
\hline 6121 & 1 & 7 & 1 & 7 & $\overline{1}$ \\
\hline$\overline{6131}$ & \begin{tabular}{l|l}
1 & \\
\end{tabular} & 2 & $\overline{\overline{1}}$ & $\overline{\overline{2}}$ & $\overline{11}$ \\
\hline 6151 & 1 & 3 & 1 & $\overline{3}$ & $\overline{1}$ \\
\hline $6161=61.101$ & 21 & 506,184 & $\overline{(1,1)}$ & 506 & $\overline{5}$ \\
\hline \multicolumn{6}{|c|}{$\begin{array}{c}x^{5}+60084263 x^{4}-28050741776 x^{3}+92072987203 x^{2} \\
-63962905415 x-1 \\
\end{array}$} \\
\hline $6161=61.101$ & 2 & 506,184 & $\bar{~} \overline{(2,1)}$ & |506 & 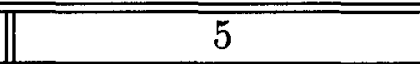 \\
\hline \multicolumn{6}{|c|}{$\begin{array}{c}x^{5}+383549085 x^{4}+26851656999175184 x^{3} \\
+170595933843787 x^{2}+218097717015 x-1\end{array}$} \\
\hline $6161=61.101$ & \begin{tabular}{|l|l}
2 & \\
\end{tabular} & 506,184 & $\overline{(3,1)}$ & \begin{tabular}{l||l||}
506 & \\
\end{tabular} & $\overline{\overline{5}}$ \\
\hline \multicolumn{6}{|c|}{$\begin{array}{l}x^{5}-4462358115542232487873 x^{4} \\
+5712618517139129990458617909278 x^{3}+ \\
3109302667860392531138 x^{2}-15092187858256756511 x-1\end{array}$} \\
\hline $6161=61.101$ & 2 & $\overline{506,184}$ & $(4,1)$ & 2506 & $\overline{5}$ \\
\hline \multicolumn{6}{|c|}{$\begin{array}{c}x^{5}+90296319386 x^{4}+89666717702616 x^{3}-5828610465955 x^{2} \\
-20992718 x-1\end{array}$} \\
\hline $6191=41.151$ & $2 \mid$ & 152,165 & $\overline{(1,1)}$ & $\mid \overline{152 \|}$ & 5 \\
\hline \multicolumn{6}{|c|}{$\begin{array}{r}x^{5}+52750436471 x^{4}-4456061787715565 x^{3} \\
+4451439214535151606 x^{2}+748096434919 x-1 \\
\end{array}$} \\
\hline $6191=41.151$ & \begin{tabular}{|l|l}
2 & \\
\end{tabular} & 152,165 & $\overline{(2,1)}$ & 152 & 5 \\
\hline \multicolumn{6}{|c|}{$\begin{array}{l}x^{5}-9039458129 x^{4}-33046346659870599 x^{3} \\
-367699858945604454 x^{2}+16175873181-1\end{array}$} \\
\hline $6191=41.151$ & \begin{tabular}{|l|l}
2 & \\
\end{tabular} & $\overline{152,165}$ & $\overline{(3,1)}$ & $\overline{152}$ & $\overline{5}$ \\
\hline \multicolumn{6}{|c|}{$\begin{array}{c}x^{5}-214292876 x^{4}-14615338251105 x^{3}-4398999954308 x^{2} \\
+62167448 x-1\end{array}$} \\
\hline $6191=41.151$ & 2 & 152,165 & $\overline{(4,1)}$ & 152 & $\overline{5}$ \\
\hline \multicolumn{6}{|c|}{$\begin{array}{c}x^{5}-534148645035637 x^{4}-1648098210672592332765888451 x^{3} \\
+16151434332005677515437803 x^{2}-11060963345340 x-1\end{array}$} \\
\hline 6211 & 1 & $\overline{2}$ & $\overline{1}$ & $\overline{22}$ & $\overline{1}$ \\
\hline 6221 & 1 & $\overline{3}$ & $\overline{1}$ & $\overline{3}$ & 1 \\
\hline 6271 & 1 & 11 & 1 & 11 & $\overline{1}$ \\
\hline
\end{tabular}




\begin{tabular}{|c|c|c|c|c|c|}
\hline$f$ & & $\left(g_{i}\right)_{i=1, \ldots, k}$ & $k-$ uple & gen & $h$ \\
\hline \multicolumn{6}{|c|}{$p(x)$} \\
\hline $6275=25.251$ & 2 & $\overline{252,26}$ & $(1,1)$ & 252 & 25 \\
\hline \multicolumn{6}{|c|}{$\begin{array}{c}x^{5}-27755254211350 x^{4}+1923441979702915 x^{3} \\
+15463594999220 x^{2}-21746540 x-1\end{array}$} \\
\hline $6275=25.251$ & 2 & 252,26 & $\overline{(2,1)}$ & $\overline{\overline{252}}$ & $\overline{25}$ \\
\hline \multicolumn{6}{|c|}{$x^{5}-82140 x^{4}+1263708125 x^{3}+604510003655 x^{2}+74145 x-1$} \\
\hline $6275=25.251$ & 2 & 252,26 & $(3,1)$ & 252 & 25 \\
\hline \multicolumn{6}{|c|}{$\begin{aligned} x^{5}+52168530 x^{4}- & 1380436499830 x^{3}-94838954675 x^{2} \\
- & 730310 x-1\end{aligned}$} \\
\hline $6275=25.251$ & \begin{tabular}{|l|l}
2 &
\end{tabular} & 252,26 & $\overline{(4,1)}$ & 252 & $275=25.11$ \\
\hline \multicolumn{6}{|c|}{$x^{5}-745 x^{4}+48820 x^{3}+56275 x^{2}+6115 x-1$} \\
\hline $6281=11.571$ & 2 & 22285,12 & $\overline{(1,1)}$ & 2285 & 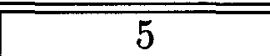 \\
\hline \multicolumn{6}{|c|}{$\begin{array}{r}x^{5}+84436018068 x^{4}+975527674877023069 x^{3} \\
+1521353541615679742983 x^{2}-9934535749576345 x-1\end{array}$} \\
\hline $6281=11.571$ & 20 & 2285,12 & $\overline{(2,1)}$ & 22285 & $\overline{5}$ \\
\hline \multicolumn{6}{|c|}{$\begin{array}{c}x^{5}-262745609 x^{4}-185301838688308 x^{3}-15311508563 x^{2} \\
+324021 x-1\end{array}$} \\
\hline $6281=11.571$ & 2 & 2285,12 & $\overline{(3,1)}$ & $\mid 2285$ & $\overline{55}$ \\
\hline \multicolumn{6}{|c|}{$\begin{array}{c}x^{5}-1437197785732 x^{4}-51100265742675535 x^{3} \\
+7955442919022556 x^{2}-280395556656 x-1 \\
\end{array}$} \\
\hline $6281=11.571$ & 2 & 2285,12 & $(4.1)$ & 2285 & $\overline{5}$ \\
\hline \multirow{2}{*}{\multicolumn{6}{|c|}{$\begin{array}{r}x^{5}-4536455891797 x^{4}-69394117995473325312215 x^{3} \\
+2595720783306189727541540639 x^{2}+9530320397143276 x-1 \\
\end{array}$}} \\
\hline & & & & & \\
\hline 6301 & \begin{tabular}{|l|l}
1 &
\end{tabular} & $\overline{10}$ & 1 & 10 & $\overline{1}$ \\
\hline 6311 & 1 & 7 & 1 & 7 & 1 \\
\hline 6361 & \begin{tabular}{l|l}
1 &
\end{tabular} & 19 & $\overline{1}$ & $\overline{19}$ & $\overline{1}$ \\
\hline 6421 & 1 & 6 & 1 & 6 & 1 \\
\hline$\overline{6451}$ & $\overline{\mid 11}$ & $\overline{\overline{3}}$ & $\overline{\overline{1}}$ & $\overline{\overline{3}}$ & $\overline{11}$ \\
\hline 6481 & 1 & 7 & 1 & 7 & 1 \\
\hline 6491 & 1 & 2 & 1 & 2 & 1 \\
\hline 6521 & 1 & 6 & 1 & 6 & $\overline{1}$ \\
\hline $6541=31.211$ & \begin{tabular}{l|l}
2 & \\
\end{tabular} & 1478,187 & $(1,1)$ & 1478 & 25 \\
\hline \multicolumn{6}{|c|}{$\begin{array}{c}x^{5}+614906443 x^{4}+1023759047692009 x^{3} \\
-14804431073599255 x^{2}-30617708600 x-1 \\
\end{array}$} \\
\hline $6541=31.211$ & 2 & 1478,187 & $\overline{(2,1)}$ & 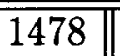 & 25 \\
\hline$x^{x}$ & & & 0 & & $06506 x-1$ \\
\hline
\end{tabular}




\begin{tabular}{|c|c|c|c|c|c|}
\hline$f$ & $k$ & $\left(g_{i}\right)_{i=1, \ldots, k}$ & $k-u p l e$ & gen & $h$ \\
\hline \multicolumn{6}{|c|}{$p(x)$} \\
\hline $6541=31.211$ & & 1478,187 & $(3,1)$ & 1478 & 25 \\
\hline \multicolumn{6}{|c|}{$\begin{array}{l}x^{5}-6739100498005 x^{4}-213706315608814024583 x^{3} \\
+3582306417082339043 x^{2}-8855265375332564 x-1\end{array}$} \\
\hline $6541=31.211$ & 2 & 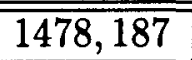 & $\overline{(4,1)}$ & 1478 & $\overline{25}$ \\
\hline \multicolumn{6}{|c|}{$\begin{array}{c}x^{5}+175988568595187950872191 x^{4} \\
-200442842075569811399671981026 x^{3} \\
-15216967152909999968589921406 x^{2}+7326947869126113 x-1\end{array}$} \\
\hline (6551 & 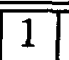 & $\overline{17}$ & 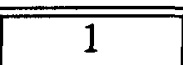 & $\overline{17}$ & $\overline{\overline{1}}$ \\
\hline$\overline{6571}$ & 1 & 3 & $\overline{1}$ & $\overline{3}$ & $\overline{1}$ \\
\hline$\overline{6581}$ & 1 & $\overline{14}$ & $\overline{1}$ & $\overline{14}$ & 11 \\
\hline \multicolumn{6}{|c|}{$\begin{array}{c}x^{5}+196423125184591289611451495207064625 x^{4} \\
-22959314992255394349858728296586145266 x^{3} \\
+13387336538069334344283562745750817074 x^{2} \\
-2560352140490106811633 x+1\end{array}$} \\
\hline $6611=11.601$ & \begin{tabular}{|l|l|}
2 & \\
\end{tabular} & 602,34 & $(1,1)$ & \begin{tabular}{|l|}
602 \\
\end{tabular} & 5 \\
\hline \multicolumn{6}{|c|}{$\begin{array}{l}x^{5}-60487611504 x^{4}-3957191640305439782 x^{3} \\
-45273568094723342063 x^{2}+23682119882 x-1\end{array}$} \\
\hline $6611=11.601$ & \begin{tabular}{|l|l|}
2 & \\
\end{tabular} & 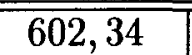 & $\overline{(2,1)}$ & \begin{tabular}{ll|}
602 \\
\end{tabular} & $\overline{55}$ \\
\hline \multicolumn{6}{|c|}{$\begin{array}{c}x^{5}+46190480447011485235263095 x^{4} \\
+149029123941115958888697755212819163758790 x^{3} \\
-96407023474274952199203638696604091164305610742 x^{2} \\
+22020659607692685330130640445625 x-1\end{array}$} \\
\hline $6611=11.601$ & & 602,34 & $\overline{(3,1)}$ & $\overline{6002}$ & 5 \\
\hline \multicolumn{6}{|c|}{$\begin{array}{c}x^{5}+256433441780 x^{4}-896695986120495466712 x^{3} \\
-104937630411923959159649521 x^{2}+193394914170512759356 x-1\end{array}$} \\
\hline $6611=11.601$ & 2 & 602,34 & $(4,1)$ & 602 & 5 \\
\hline \multicolumn{6}{|c|}{$x^{5}-29685954 x^{4}-100907877 x^{3}-104358977 x^{2}-32627933 x-1$} \\
\hline 6661 & 1 & 6 & 1 & 6 & 1 \\
\hline 6691 & 1 & 2 & 1 & 2 & 1 \\
\hline 6701 & 1 & 2 & 1 & 2 & 1 \\
\hline 6761 & 1 & 3 & 1 & $\overline{3}$ & 1 \\
\hline $6775=25.271$ & 2 & 272,26 & $(1,1)$ & 272 & 5 \\
\hline \multicolumn{6}{|c|}{$\begin{array}{c}x^{5}-7221213700 x^{4}+156260105415 x^{3}+4874673232445 x^{2} \\
-240934994415 x-1\end{array}$} \\
\hline $6775=25.271$ & 2 & 272,26 & $(2,1)$ & 272 & 5 \\
\hline \multicolumn{6}{|c|}{$\begin{array}{c}x^{5}+169031806950 x^{4}+312707038306900136690 x^{3} \\
-200497683435968881505 x^{2}-282045273284640 x-1 \\
\end{array}$} \\
\hline
\end{tabular}




\begin{tabular}{|c|c|c|c|c|}
\hline$f$ & $\begin{array}{l}{ }_{i} \\
\left(g_{i}\right)_{i=1, \ldots, k}\end{array}$ & $k-u p l e$ & $g e n$ & $h$ \\
\hline \multicolumn{5}{|c|}{$p(x)$} \\
\hline \begin{tabular}{l|l|l}
$6775=25.271$ & 2
\end{tabular} & $\overline{\overline{272,26}}$ & $\overline{(3,1)}$ & \begin{tabular}{|l|}
272 \\
\end{tabular} & $\overline{5}$ \\
\hline \multicolumn{5}{|c|}{$\begin{array}{c}x^{5}+8438306586275 x^{4}+15811513700173582894698690 x^{3} \\
-1336864969219470161701257272396130 x^{2}-96777249836067715 x-1\end{array}$} \\
\hline \begin{tabular}{|l|l}
$6775=25.271$ & 2
\end{tabular} & $\overline{272,26}$ & $\overline{(4,1)}$ & 272 & $\overline{5}$ \\
\hline \multicolumn{5}{|c|}{$\begin{array}{l}x^{5}-232246605700 x^{4}+8351446117955891065 x^{3} \\
+8711856154450720 x^{2}+433034370160 x-1\end{array}$} \\
\hline$\overline{\overline{6781}}$ & $\overline{22}$ & $\overline{1} 1$ & $\overline{22}$ & $\overline{1}$ \\
\hline 6791 & 7 & 1 & 7 & 1 \\
\hline (6841 & $\overline{22}$ & $\overline{1}$ & $\overline{22}$ & $\overline{1}$ \\
\hline 6871 & 3 & 1 & $\overline{3}$ & $\overline{1}$ \\
\hline (6911 & $\overline{7}$ & $\overline{1}$ & $\overline{7}$ & $\overline{11}$ \\
\hline$\overline{c 6961}$ & $\overline{13}$ & 1 & $\overline{13}$ & $\overline{1}$ \\
\hline$\overline{\overline{6971}}$ & $\overline{2}$ & $\overline{1}$ & $\overline{2}$ & 1 \\
\hline \begin{tabular}{l|l|}
$6941=11.631$ & 2
\end{tabular} & 1894,12 & $\overline{(1,1)}$ & $|\overline{\mid l 1894}| \mid$ & $\overline{5}$ \\
\hline \multicolumn{5}{|c|}{$\begin{array}{r}x^{5}-93656442260433781 x^{4}-978770726523793476923101862 x^{3} \\
-46621910847584118509741582058 x^{2}-2063920466942543347643 x-1\end{array}$} \\
\hline \begin{tabular}{|l|l|}
$6941=11.631$ & 2 \\
\end{tabular} & 1894,12 & $(2,1)$ & 1894 & 5 \\
\hline \multicolumn{5}{|c|}{$\begin{array}{l}x^{5}+43048050830991 x^{4}-2550548095099740794704682 x^{3} \\
+14305998862153190036745157 x^{2}+94092615475497 x-1\end{array}$} \\
\hline \begin{tabular}{|l|l|}
$6941=11.631$ & 2 \\
\end{tabular} & \begin{tabular}{|l|}
1894,12 \\
\end{tabular} & $(3,1)$ & 1894 & 5 \\
\hline \multicolumn{5}{|c|}{$\begin{array}{c}x^{5}-46085218 x^{4}-13126557579 x^{3}+571013912363 x^{2} \\
+2205014634219 x-1\end{array}$} \\
\hline \begin{tabular}{|l|l|}
$6941=11.631$ & 2 \\
\end{tabular} & 1894,12 & $\overline{(4,1)}$ & |1894 || & 5 \\
\hline \multicolumn{5}{|c|}{$\begin{array}{c}x^{5}-12022426813189972 x^{4}+35387540895365378245680789511 x^{3} \\
+3012115297054679098688290854077564 x^{2} \\
-39834486955864175795145452 x-1\end{array}$} \\
\hline 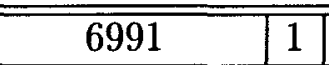 & $\overline{6}$ & $\overline{1}$ & 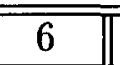 & $\overline{1}$ \\
\hline$\overline{7001}$ & 3 & 1 & $\overline{3}$ & $\overline{1}$ \\
\hline \begin{tabular}{|l|l|}
$7025=25.281$ & 2 \\
\end{tabular} & 563,26 & $(1,1)$ & 563 & 5 \\
\hline \multicolumn{5}{|c|}{$\begin{array}{c}x^{5}-526684623763525 x^{4}-7732356332152028530233785 x^{3} \\
-44967399719215436155 x^{2}+25149964810 x-1\end{array}$} \\
\hline \begin{tabular}{|l|l|}
$7025=25.281$ & 2 \\
\end{tabular} & 563,26 & $\overline{(2,1)}$ & $\overline{5633}$ & $\overline{5}$ \\
\hline & +61614751 & $x^{4}+1$ & $720777^{\prime}$ & $66965 x^{3}$ \\
\hline
\end{tabular}




\begin{tabular}{|c|c|c|c|c|c|}
\hline$f$ & & $\left(g_{i}\right)_{i=1, \ldots, k}$ & $k$-uple & gen & $h$ \\
\hline \multicolumn{6}{|c|}{$p(x)$} \\
\hline $7025=25.281$ & \begin{tabular}{|l|l}
2 & \\
\end{tabular} & $\overline{563,26}$ & $\overline{(3,1)}$ & 563 & $\overline{5}$ \\
\hline \multirow{2}{*}{\multicolumn{6}{|c|}{$\begin{array}{l}x^{5}+154814262947698362075 x^{4}+4339984100657763657 \\
2459585151871690 x^{3}+2792850702468992360003 \\
2721220881860870 x^{2}-17280527492604181486315 x-1\end{array}$}} \\
\hline & & & & & \\
\hline $7025=25.281$ & $\overline{22}$ & 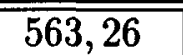 & $\overline{(4,1)}$ & 563 & $\overline{55}$ \\
\hline \multicolumn{6}{|c|}{$\begin{array}{c}x^{5}+7868975 x^{4}-155272024385 x^{3}-95588261315980 x^{2} \\
-51031741465 x-1\end{array}$} \\
\hline $7051=11.641$ & 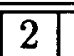 & $\overline{11283,12}$ & $\overline{(1,1)}$ & 1283 & $\overline{\overline{5}}$ \\
\hline \multirow{2}{*}{\multicolumn{6}{|c|}{$\begin{array}{c}x^{5}+14126843431966092849 x^{4}-629957648898356919 \\
2468325846888661123 x^{3}+29215894218214236615105 \\
575323746906075202 x^{2}+2024306807385606756046851 x-1\end{array}$}} \\
\hline & & & & & \\
\hline $7051=11.641$ & \begin{tabular}{|l|}
2 \\
\end{tabular} & $\overline{1283,12}$ & $\overline{(2,1)}$ & |1283 & $\overline{5}$ \\
\hline \multirow{2}{*}{\multicolumn{6}{|c|}{$\begin{array}{l}x^{5}+45997854239292 x^{4}+3808959110676225841573 x^{3} \\
-16874898401741215377902 x^{2}-452676162496 x-1\end{array}$}} \\
\hline & & & & & \\
\hline $7051=11.641$ & \begin{tabular}{|l|l}
2 & \\
\end{tabular} & \begin{tabular}{ll|}
1283,12 \\
\end{tabular} & $(3,1)$ & 1283 & $\overline{5}$ \\
\hline \multicolumn{6}{|c|}{$\begin{aligned} x^{5}+9395933260 x^{4}- & 1666021036008663 x^{3}+407606293581 x^{2} \\
& -19526537 x-1\end{aligned}$} \\
\hline $7051=11.641$ & $\overline{22}$ & 1283,12 & $\overline{(\overline{(4,1)}}$ & 1283 & $\overline{5}$ \\
\hline \multicolumn{6}{|c|}{$\begin{array}{r}x^{5}+90429143884562 x^{4}+2203309979373504283 x^{3} \\
-11231443712025425394492 x^{2}-13086612624566 x-1 \\
\end{array}$} \\
\hline 7121 & 1 & $\overline{3}$ & $\overline{11}$ & $\overline{3}$ & $\overline{1}$ \\
\hline 7151 & 1 & 7 & 1 & 7 & 1 \\
\hline $7171=71.101$ & 2 & 102,72 & $\overline{(1,1)}$ & 102 & $\overline{5}$ \\
\hline \multirow{4}{*}{\multicolumn{6}{|c|}{$\begin{array}{c}x^{5}-38078801025544460332585 x^{4} \\
-7528495331870354339022286315035569 x^{3} \\
-3265447724932472592706656692041804 x^{2} \\
-115312912352936797265 x-1 \\
\end{array}$}} \\
\hline & & & & & \\
\hline & & & & & \\
\hline & & & & & \\
\hline $7171=71.101$ & & 102,72 & $\overline{(2,1)}$ & $\overline{102}$ & $\overline{5}$ \\
\hline \multirow{2}{*}{\multicolumn{6}{|c|}{$\begin{array}{c}x^{5}-158257017 x^{4}+5017041158843294 x^{3} \\
-37726546298573054109787 x^{2}-2055498579615 x-1\end{array}$}} \\
\hline & & & & & \\
\hline $7171=71.101$ & \begin{tabular}{l|l}
2 & \\
\end{tabular} & $\overline{102,72}$ & $\overline{(\overline{(3,1)}}$ & 102 & $\overline{5}$ \\
\hline \multicolumn{6}{|c|}{$\begin{array}{c}x^{5}-3485324 x^{4}+13298067698 x^{3}+5470029956585 x^{2} \\
-627525756 x-1 \\
\end{array}$} \\
\hline$\overline{7171=71.101}$ & $\overline{\overline{2}}$ & $\overline{102,72}$ & $\overline{(4,1)}$ & $102 \pi$ & $\overline{\overline{5}}$ \\
\hline \multirow{2}{*}{\multicolumn{6}{|c|}{$\begin{array}{c}x^{5}-108745489822575417 x^{4}+1755472658324687358141835444 \\
758 x^{3}+212551570060735386794600951366053582266 x^{2} \\
-524222716866636913752935319 x-1\end{array}$}} \\
\hline & & & & & \\
\hline 7211 & 1 & 2 & 1 & 2 & 1 \\
\hline
\end{tabular}




\begin{tabular}{|c|c|c|c|c|}
\hline$f$ & \begin{tabular}{l|l} 
& $\left(g_{i}\right)_{i=1, \ldots, k}$ \\
\end{tabular} & $k-$ uple & gen & $h$ \\
\hline \multicolumn{5}{|c|}{$p(x)$} \\
\hline \begin{tabular}{l|l|l}
$7271=11.661$ & 2
\end{tabular} & $\overline{662,23}$ & $\overline{(1,1)}$ & \begin{tabular}{|l||}
662 \\
\end{tabular} & 25 \\
\hline \multicolumn{5}{|c|}{$\begin{array}{l}x^{5}-436505795818 x^{4}+133718059679871933 x^{3} \\
-1064675198096834605 x^{2}-2311679559 x-1\end{array}$} \\
\hline \begin{tabular}{l|l}
$7271=11.661$ & 2
\end{tabular} & 662,23 & $(2,1)$ & \begin{tabular}{|l||}
662 \\
\end{tabular} & 25 \\
\hline \multicolumn{5}{|c|}{$\begin{array}{c}x^{5}-4809867296 x^{4}+127030508489022113 x^{3} \\
+5511491411459323 x^{2}-559373847 x-1\end{array}$} \\
\hline 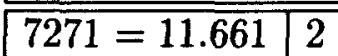 & \begin{tabular}{l|l}
662,23 \\
\end{tabular} & $\overline{(\overline{(3,1)}}$ & \begin{tabular}{|l||}
662 \\
\end{tabular} & $\overline{\overline{25}}$ \\
\hline \multicolumn{5}{|c|}{$\begin{array}{c}x^{5}-2143612397884 x^{4}-529540510372431747026820 x^{3} \\
+6557221665097928135 x^{2}-5743287957756 x-1\end{array}$} \\
\hline \begin{tabular}{l|l|l}
$7271=11.661$ & 2
\end{tabular} & 662,23 & $(4,1)$ & \begin{tabular}{|l||}
662 \\
\end{tabular} & 25 \\
\hline \multicolumn{5}{|c|}{$\begin{array}{c}x^{5}+70765417123359 x^{4}-31506955876141232242164194 x^{3} \\
+1527117812296305685955997312802 x^{2}+4728009437907585185 x-1 \\
\end{array}$} \\
\hline 7321 & 7 & 1 & 7 & 1 \\
\hline 7331 & 2 & 1 & 2 & 1 \\
\hline 7351 & 6 & 1 & 6 & $\overline{1}$ \\
\hline 7411 & 2 & 1 & 2 & 1 \\
\hline \begin{tabular}{l|l|l}
7421 & $=41.181$ & 2 \\
\end{tabular} & 363,83 & $(1,1)$ & 363 & $\overline{5}$ \\
\hline \multicolumn{5}{|c|}{$\begin{array}{r}x^{5}-838354796 x^{4}-1001496762430198 x^{3} \\
-355864285737052443 x^{2}+2928186715 x-1 \\
\end{array}$} \\
\hline \begin{tabular}{l|l|}
$7421=41.181$ & 2 \\
\end{tabular} & 363,83 & $(2,1)$ & 363 & 5 \\
\hline \multicolumn{5}{|c|}{$\begin{array}{r}x^{5}+7064090000 x^{4}+124043111434154 x^{3} \\
-2653133031716395171 x^{2}+283074142588 x-1 \\
\end{array}$} \\
\hline \begin{tabular}{l|l|}
$7421=41.181$ & \\
\end{tabular} & 363,83 & $\overline{(3,1)}$ & 363 & $\overline{55}$ \\
\hline \multicolumn{5}{|c|}{$x^{5}-790378 x^{4}-1007725843 x^{3}+137488130091 x^{2}+918327 x-1$} \\
\hline \begin{tabular}{|l|l|}
$7421=41.181$ & 2 \\
\end{tabular} & 363,83 & $(4,1)$ & 363 & 5 \\
\hline \multicolumn{5}{|c|}{$\begin{array}{c}x^{5}+327099585212919893051 x^{4}+95812231394988163213137354 x^{3} \\
+5415568472826679098470 x^{2}-82460711211 x-1\end{array}$} \\
\hline 7451 & 2 & 1 & 2 & 1 \\
\hline \begin{tabular}{|l|l|}
$7471=31.241$ & 2 \\
\end{tabular} & 724,280 & $(1,1)$ & 724 & 5 \\
\hline \multicolumn{5}{|c|}{$\begin{array}{c}x^{5}-765653778980786605810 x^{4} \\
-4629189702619227692803856712676857845 x^{3} \\
-586025841524689563386171909296439677 x^{2} \\
-1093090177239919585495521077 x-1 \\
\end{array}$} \\
\hline \begin{tabular}{|l|l|}
$7471=31.241$ & 2 \\
\end{tabular} & 724,280 & $(2,1)$ & 724 & 5 \\
\hline$x^{5}+155$ & $58162 x^{4}-$ & $\begin{array}{l}57444868 \\
84342 x-\end{array}$ & $03 x^{3}$ & 3863906 \\
\hline
\end{tabular}




\begin{tabular}{|c|c|c|c|c|c|}
\hline$f$ & $k$ & $\left(g_{i}\right)_{i=1, \ldots, k}$ & $k$-uple & gen & $h$ \\
\hline \multicolumn{6}{|c|}{$p(x)$} \\
\hline $7471=31.241$ & \begin{tabular}{|l|l|}
2 & \\
\end{tabular} & $\overline{7724,280}$ & $(3,1)$ & 724 & $\overline{5}$ \\
\hline \multicolumn{6}{|c|}{$\begin{array}{c}x^{5}+13110003582 x^{4}+142339256734848264 x^{3} \\
+24286190033833 x^{2}-40935770 x-1 \\
\end{array}$} \\
\hline $7471=31.241$ & \begin{tabular}{|l|l|}
2 \\
\end{tabular} & $\overline{7724,280}$ & $\overline{(4,1)}$ & $\mid 724$ & $\overline{5}$ \\
\hline \multicolumn{6}{|c|}{$\begin{aligned} x^{5}-5098399799 x^{4} & +867446694986 x^{3}+291216750189687 x^{2} \\
& -43001628787 x-1\end{aligned}$} \\
\hline$\overline{77481}$ & $\begin{array}{ll}1 \\
\end{array}$ & 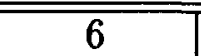 & $\bar{~} 1$ & 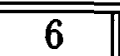 & 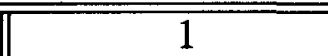 \\
\hline 7541 & 1 & 2 & 1 & $\overline{2}$ & $\overline{1}$ \\
\hline$\overline{7561}$ & $\overline{1}$ & $\overline{13}$ & $\overline{1}$ & 13 & $\overline{1}$ \\
\hline$\overline{7591}$ & 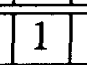 & $\overline{6}$ & $\overline{1}$ & $\overline{6}$ & 1 \\
\hline$\overline{77601=11.691}$ & 2 & $=$ & $\overline{(1,1)}$ & $\mid \overline{|c|||}$ & $\overline{5}$ \\
\hline \multicolumn{6}{|c|}{$\begin{array}{c}x^{5}+1019911 x^{4}-8130225834 x^{3}+14286989549754 x^{2} \\
+363286345 x-1\end{array}$} \\
\hline $7601=11.691$ & 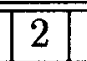 & (1383,12 & $\overline{(2,1)}$ & 1383 & $\overline{5}$ \\
\hline \multicolumn{6}{|c|}{$\begin{array}{c}x^{5}-228838319367 x^{4}-130396978764124541254 x^{3} \\
+42528934592185620379 x^{2}-1582369529561707 x-1 \\
\end{array}$} \\
\hline $7601=11.691$ & 2 & $\overline{1383,12}$ & $\overline{(3,1)}$ & 1383|| & 5 \\
\hline \multicolumn{6}{|c|}{$\begin{array}{c}x^{5}-102409355365102226943980575 x^{4} \\
-8519185198634870186671764906446 x^{3} \\
2324156936621973915671 x^{2}-393952893737 x-1 \\
\end{array}$} \\
\hline $7601=11.691$ & 2 & 1383,12 & $\overline{(4,1)}$ & \begin{tabular}{|c||}
1383 \\
$\mid$
\end{tabular} & 5 \\
\hline \multicolumn{6}{|c|}{$\begin{array}{c}x^{5}-74490617679777913 x^{4}-100980407716646656981224624330 x^{3} \\
-5748226627502579231262730471555989671302 x^{2} \\
+61569467192332331878089 x-1\end{array}$} \\
\hline$\overline{7621}$ & 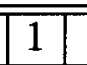 & $\overline{22}$ & $\overline{1}$ & 2 & $\overline{1}$ \\
\hline 7681 & 11 & 17 & $\overline{1}$ & $\overline{17}$ & 1 \\
\hline 7691 & 1 & 2 & 1 & 2 & 1 \\
\hline $711=11.701$ & \begin{tabular}{|l|l|}
2 & \\
\end{tabular} & 1403,12 & $(1,1)$ & 1403 & 55 \\
\hline \multicolumn{6}{|c|}{$\begin{array}{r}x^{5}+12461577167746527 x^{4}+8001176732630552638 x^{3} \\
-812778251973632120798 x^{2}+15416303877450177 x-1\end{array}$} \\
\hline $711=11.701$ & \begin{tabular}{|l|l}
2 & \\
\end{tabular} & 1403,12 & $\overline{(2,1)}$ & 1403 & 5 \\
\hline \multicolumn{6}{|c|}{$\begin{array}{c}x^{5}-37133041573 x^{4}-3548364985860892509 x^{3} \\
+20007626965751576528 x^{2}+65512351726429 x-1 \\
\end{array}$} \\
\hline $7711=11.701$ & \begin{tabular}{l|l}
2 & \\
2
\end{tabular} & 1403,12 & $\overline{(3,1)}$ & 1403 & $\overline{5}$ \\
\hline $\begin{array}{r}x^{5}+1016 \\
+141710148 \\
\end{array}$ & & $\begin{array}{l}298597 x^{4} \\
238111684\end{array}$ & $\begin{array}{r}+55422 \\
16872 x^{2} \\
\end{array}$ & 64178 & $\begin{array}{l}0531773977 x^{3} \\
848471289 x-1\end{array}$ \\
\hline
\end{tabular}




\begin{tabular}{|c|c|c|c|c|}
\hline$k$ & $\left(g_{i}\right)_{i=1, \ldots, k}$ & $k$-uple & gen & $n$ \\
\hline \multicolumn{5}{|c|}{$p(x)$} \\
\hline \begin{tabular}{|l|l|}
$711=11.701$ & 2 \\
\end{tabular} & 1403,12 & $\overline{(4,1)}$ & 1403 & 5 \\
\hline \multicolumn{5}{|c|}{$\begin{array}{c}x^{5}+7097241031011 x^{4}+6701214516902480670 x^{3} \\
+1056762535143339664184317 x^{2}-95387863307261 x-1\end{array}$} \\
\hline$\overline{7741}$ & $\overline{7}$ & $\overline{1}$ & 7 & $\overline{1.1}$ \\
\hline \begin{tabular}{|l|l|}
$7775=25.311$ & 2
\end{tabular} & $\overline{312,76}$ & $\overline{(1,1)}$ & 312 & $\overline{5}$ \\
\hline \multicolumn{5}{|c|}{$\begin{array}{l}x^{5}+4925208855 x^{4}+1144227744355120 x^{3} \\
-11994529778075 x^{2}+5314597665 x-1\end{array}$} \\
\hline 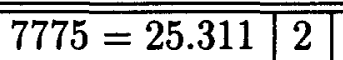 & $\overline{312,76}$ & $\overline{(2,1)}$ & 312 & $\overline{5}$ \\
\hline \multicolumn{5}{|c|}{$\begin{array}{c}x^{5}-160014493567997728575917306019625014745 x^{4} \\
-1212529331544066885299101711804486942380233193270807337130 x^{3} \\
+184177706154842698977569510698223196966669034307422341613803 \\
887450 x^{2}-62126011215380118074926778192915525928592542935 x-1\end{array}$} \\
\hline $\begin{array}{l}7775=25.311 \\
\end{array}$ & $\overline{312,76}$ & $\overline{(3,1)}$ & \begin{tabular}{ll|}
312 \\
\end{tabular} & $\overline{5}$ \\
\hline \multicolumn{5}{|c|}{$\begin{array}{c}x^{5}-832909156570 x^{4}-4704121217276655 x^{3} \\
+13018342556101175 x^{2}+827194215 x-1 \\
\end{array}$} \\
\hline \begin{tabular}{|l|l|}
$7775=25.311$ & 2 \\
\end{tabular} & 312,76 & $\overline{(4,1)}$ & 312 & 5 \\
\hline \multicolumn{5}{|c|}{$\begin{array}{c}x^{5}+84251963495705 x^{4}+139480454097620 x^{3} \\
+3410176417775 x^{2}+114864265 x-1\end{array}$} \\
\hline \begin{tabular}{|l|l|}
$7781=31.251$ & 2 \\
\end{tabular} & \begin{tabular}{|l|}
1005,280 \\
\end{tabular} & (1,1) & 1005 & $\overline{\overline{5}}$ \\
\hline \multicolumn{5}{|c|}{$\begin{array}{c}x^{5}+40598615883 x^{4}+1398552863379785149 x^{3} \\
-291243520100541963782 x^{2}+91187701834630119575 x-1\end{array}$} \\
\hline \begin{tabular}{l|l|}
$781=31.251$ & 2 \\
7
\end{tabular} & 1005,280 & $\overline{(2,1)}$ & 1005 & $\overline{5}$ \\
\hline \multicolumn{5}{|c|}{$\begin{aligned} x^{5}+6527730774676 x^{4}- & 2556208894017905 x^{3}+398384657495 x^{2} \\
& +6389699 x-1\end{aligned}$} \\
\hline \begin{tabular}{|l|l|}
$781=31.251$ & 2 \\
\end{tabular} & 1005,280 & $(3,1)$ & 1005 & 5 \\
\hline \multicolumn{5}{|c|}{$\begin{array}{l}x^{5}+9615917280132134909 x^{4} \\
71375684021484931436925116285 x^{3} \\
062898741154961049309333828696 x^{2} \\
+965686784246497394443 x-1 \\
\end{array}$} \\
\hline \begin{tabular}{|l|l|}
$781=31.251$ & 2 \\
\end{tabular} & 101005,280 & $\overline{(4,1)}$ & $\mid$ & $\overline{\overline{5}}$ \\
\hline \multicolumn{5}{|c|}{$\begin{array}{l}x^{5}+1396794076 x^{4}-17264095858816460 x^{3} \\
+167598581625395 x^{2}+860004304 x-1\end{array}$} \\
\hline \begin{tabular}{|l|l|}
$831=41.191$ & 2 \\
8
\end{tabular} & 192,42 & $\overline{(1,1)}$ & 192 & $\overline{\overline{25}}$ \\
\hline \multicolumn{5}{|c|}{$\begin{array}{c}x^{5}-1933491423344729923537669473 x^{4} \\
+170455757748945846153613428691454 x^{3} \\
-191304760895067032450023984103582 x^{2}-5622238420357263\end{array}$} \\
\hline \begin{tabular}{|l|l|}
$7831=41.191$ & 2
\end{tabular} & 192,42 & $\overline{(2,1)}$ & $\overline{192}$ & $\overline{\overline{25}}$ \\
\hline $\begin{array}{r}5+25564 \\
+360606 \\
\end{array}$ & $\begin{array}{l}440588887 x^{4} \\
1026757402\end{array}$ & $\begin{array}{l}+6144 \\
49 x^{2}+\end{array}$ & 24502 & $\begin{array}{l}4245673137 x^{3} \\
138116 x-1\end{array}$ \\
\hline
\end{tabular}




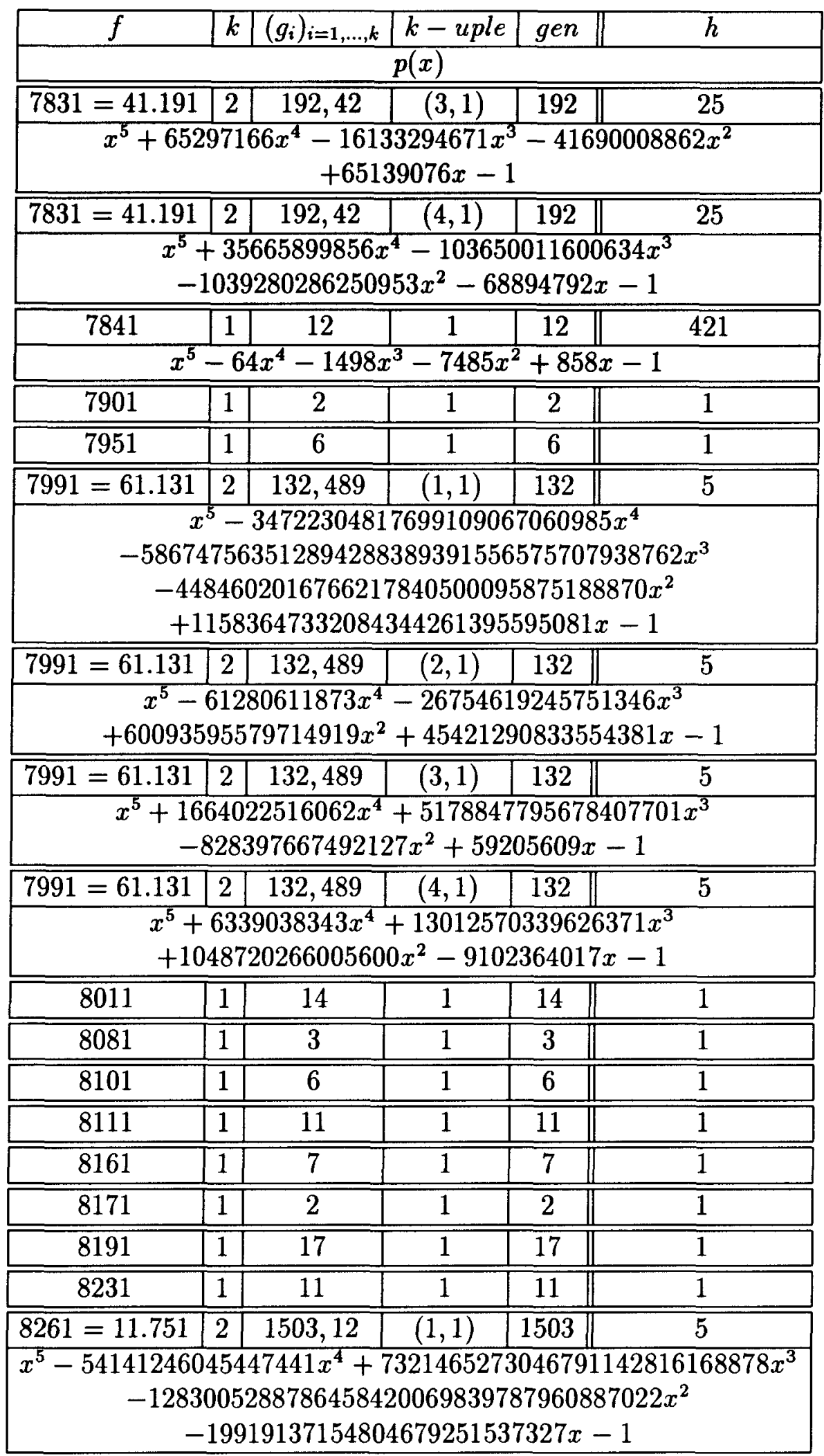




\begin{tabular}{|c|c|c|c|c|c|}
\hline$f$ & $k$ & $\left(g_{i}\right)_{i=1, \ldots, k}$ & $k-u p l e$ & gen & $h$ \\
\hline \multicolumn{6}{|c|}{$p(x)$} \\
\hline $8261=11.751$ & 2 & 1503,12 & $(2,1)$ & 1503 & 5 \\
\hline \multicolumn{6}{|c|}{$\begin{array}{c}x^{5}+1133682949174226538 x^{4}-11087406782848694864185 x^{3} \\
+32154596461277806849 x^{2}-2387156436538959 x-1\end{array}$} \\
\hline $8261=11.751$ & \begin{tabular}{|l|}
2 \\
\end{tabular} & 1503,12 & $(3,1)$ & 1503 & 5 \\
\hline \multicolumn{6}{|c|}{$\begin{array}{c}x^{5}+185885200907 x^{4}-2832014033124231113 x^{3} \\
+1134230052754829110 x^{2}+7021742458609915 x-1 \\
\end{array}$} \\
\hline $8261=11.751$ & 2 & 1503,12 & $\overline{(4,1)}$ & 1503 & $\overline{5}$ \\
\hline \multicolumn{6}{|c|}{$\begin{array}{l}x^{5}+4750640498 x^{4}+8392673004627787 x^{3} \\
+14035597461351458 x^{2}-7250313958 x-1 \\
\end{array}$} \\
\hline $8275=25.331$ & 2 & 663,101 & $(1,1)$ & 663 & 5 \\
\hline \multicolumn{6}{|c|}{$\begin{array}{r}x^{5}-63856927450 x^{4}-33623370611860 x^{3} \\
-505475122158655 x^{2} \\
+4042236482860 x-1 \\
\end{array}$} \\
\hline $8275=25.331$ & 2 & 663,101 & $\overline{(2,1)}$ & 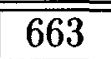 & $\overline{5}$ \\
\hline \multicolumn{6}{|c|}{$\begin{array}{r}x^{5}-145694947975 x^{4}-31798470402017994260 x^{3} \\
-10177088406243321055 x^{2}-1175605640701015 x-1 \\
\end{array}$} \\
\hline $8275=25.331$ & 21 & |663,101 & $(3,1)$ & 6663 & $\overline{5}$ \\
\hline \multicolumn{6}{|c|}{$\begin{array}{l}x^{5}+1285986150692647175 x^{4} \\
5493358024277103384089610 x^{3} \\
19867604230 x^{2}+48595211804814872585 x-1 \\
\end{array}$} \\
\hline $8275=25.331$ & 2 & 663,101 & $(4,1)$ & 663 & 5 \\
\hline \multicolumn{6}{|c|}{$\begin{array}{c}x^{5}+7370270275198364400 x^{4}-2331493073434711813885 x^{3} \\
+86613539772282898120 x^{2}+14255520846163710 x-1 \\
\end{array}$} \\
\hline 8291 & 1 & 2 & 1 & 2 & 1 \\
\hline 8311 & 1 & 3 & 1 & $\overline{3}$ & 1 \\
\hline $8371=11.761$ & 2 & 2284,12 & $(1,1)$ & 2284 & 5 \\
\hline \multicolumn{6}{|c|}{$\begin{array}{c}x^{5}+647983764314427 x^{4}-118666151682787 x^{3} \\
-1371869029027 x^{2}-2756376 x-1 \\
\end{array}$} \\
\hline $8371=11.761$ & $\overline{2}$ & $\overline{2284,12}$ & $\overline{(2,1)}$ & 2284 & 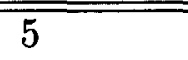 \\
\hline \multicolumn{6}{|c|}{$\begin{array}{r}x^{5}-47528406619692 x^{4}-499280153975632789501 x^{3} \\
-629976233082936170 x^{2}+247020407497406 x-1 \\
\end{array}$} \\
\hline $8371=11.761$ & 2 & 2284,12 & $(3,1)$ & 2284 & 5 \\
\hline \multicolumn{6}{|c|}{$\begin{array}{c}x^{5}-17187520 x^{4}-8218127285 x^{3}+485777242102 x^{2} \\
+2086477804 x-1 \\
\end{array}$} \\
\hline $371=11.761$ & 2 & 2284,12 & $(4,1)$ & 2284 & 5 \\
\hline \multicolumn{6}{|c|}{$\begin{array}{c}x^{5}-47621558380234591539918569 x^{4} \\
+1938472900169689712353355695135797158 x^{3} \\
+98203346678022308055972984864816746 x^{2} \\
+133196349271850997214693593 x-1 \\
\end{array}$} \\
\hline
\end{tabular}




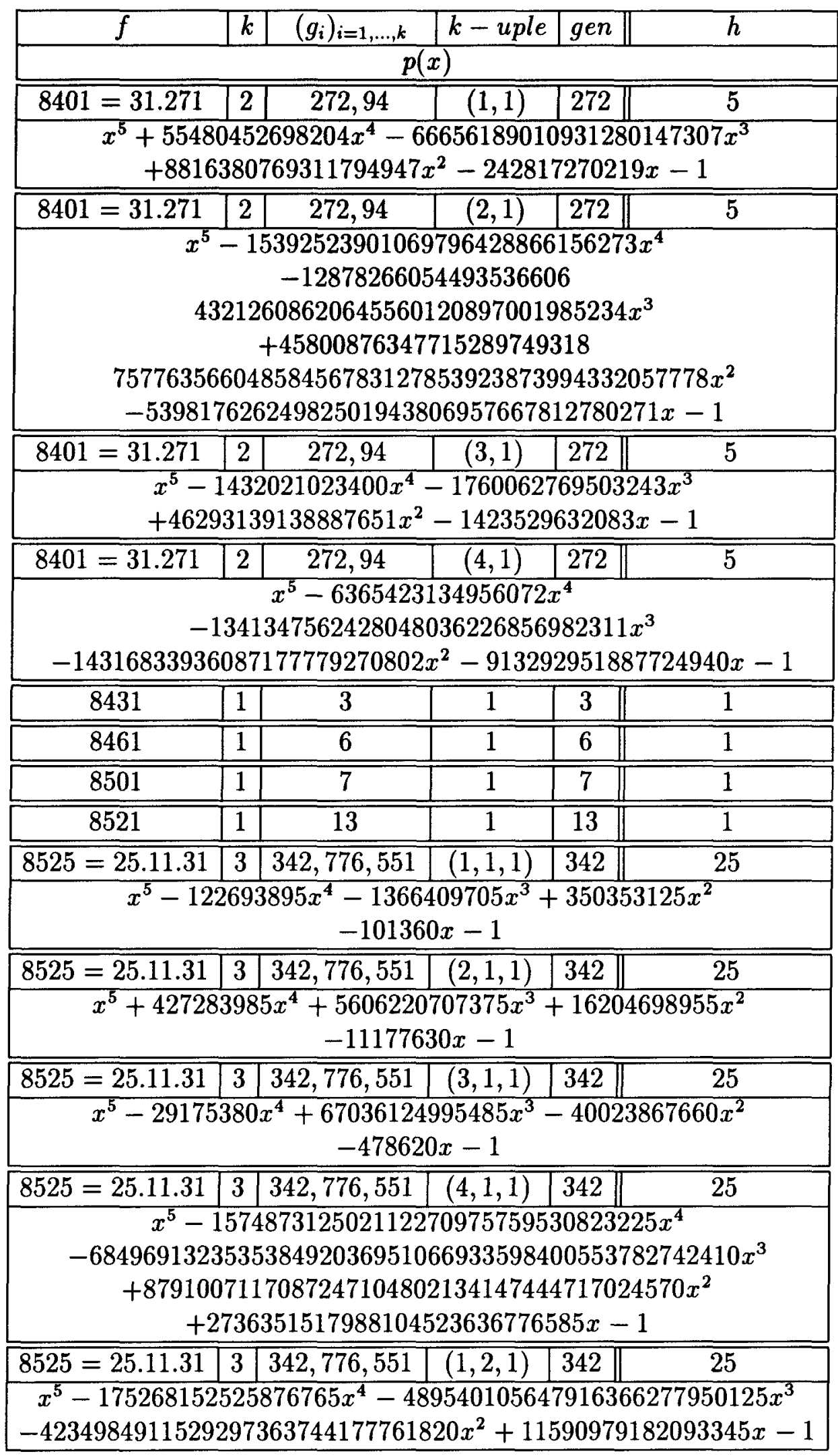




\begin{tabular}{|c|c|c|c|c|c|}
\hline J & & $\left(g_{i}\right)_{i=1}$ & & & \\
\hline \multicolumn{6}{|c|}{$p(x)$} \\
\hline إ.31 & & $342,776,551$ & $(2,2,1)$ & $\overline{342}$ & 20 \\
\hline \multicolumn{6}{|c|}{$\begin{array}{c}x^{5}-411196825312997025 x^{4}+20123750719709388934712758590 x \\
-2261305337076218940818619230 x^{2}-3456186917495615 x-1\end{array}$} \\
\hline $525=25.11 .31$ & \begin{tabular}{l|l}
3 &
\end{tabular} & $\overline{342,776,551}$ & $(3,2,1)$ & $\mid 342$ & 25 \\
\hline \multicolumn{6}{|c|}{$x^{5}+3555 x^{4}-10797880 x^{3}+5616133675 x^{2}-159679835 x-1$} \\
\hline $25=25.11 .31$ & 3 & $\overline{342,776,551}$ & $\overline{(4,2,1)}$ & 342 & 25 \\
\hline \multicolumn{6}{|c|}{$\begin{array}{c}x^{5}-94408505 x^{4}+68940181358160 x^{3}+1439452044006465 x^{2} \\
-123640954395 x-1 \\
\end{array}$} \\
\hline $525=25.11 .31$ & 3 & $\overline{342,776,551}$ & $(1,3,1)$ & 342 & 25 \\
\hline \multicolumn{6}{|c|}{$\begin{array}{c}x^{5}-74465850009060 x^{4}-425610622301544653070 x^{3} \\
-53764906259530077841967865 x^{2}+15783132179450 x-1\end{array}$} \\
\hline $525=25.11 .31$ & \begin{tabular}{l|l}
3 &
\end{tabular} & $342,776,551$ & (2,3,1) & 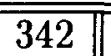 & $\overline{25}$ \\
\hline \multicolumn{6}{|c|}{$\begin{array}{l}x^{5}+95026433196015 x^{4}-28117658675439009383139845 x^{3} \\
+12200995605028308499210 x^{2}-435756349382047525 x-1 \\
\end{array}$} \\
\hline $525=25.11 .31$ & & & & $\overline{342}$ & $2 v$ \\
\hline \multicolumn{6}{|c|}{$\begin{array}{c}x^{5}-2123242224137636842960 x^{4} \\
-12665716405470830993031027740795 x^{3} \\
-685673560768622506105874815 x^{2}+8659365523737337975 x-1\end{array}$} \\
\hline$\overline{525=25.11 .31}$ & 3 & $\overline{\overline{342,776,551}}$ & 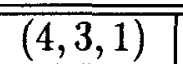 & 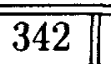 & $\overline{25}$ \\
\hline \multicolumn{6}{|c|}{$\begin{aligned} & x^{5}+6944622440 x^{4}-224845393938245 x^{3}-8498546814490 x^{2} \\
&+ 5887850 x-1 \\
&\end{aligned}$} \\
\hline $525=25.11 .31$ & & $\overline{342,776,551}$ & $\overline{(1,4,1)}$ & $342 \|$ & 25 \\
\hline \multicolumn{6}{|c|}{$x^{5}+3529495 x^{4}-595820890 x^{3}+923058090 x^{2}+160505 x-$} \\
\hline $25=25.11 .31$ & & $342,776,551$ & $\overline{(2,4,1)}$ & $\overline{342}$ & 25 \\
\hline \multicolumn{6}{|c|}{$x^{5}-16470 x^{4}-75155 x^{3}+8316675 x^{2}+57332815 x-1$} \\
\hline $25=25.11 .31$ & $\overline{3}$ & $342,776,551$ & $\overline{(3,4,1)}$ & $\overline{342}$ & $\overline{25}$ \\
\hline \multicolumn{6}{|c|}{$\begin{array}{c}x^{5}-183635997627625 x^{4}-487367092784379522283517210 x^{3} \\
+1687146866242668386212970 x^{2}-233145272474215 x-1 \\
\end{array}$} \\
\hline $5=25.11 .31$ & $\overline{3}$ & $342,776,551$ & $(4,4,1)$ & 342 & 25 \\
\hline \multicolumn{6}{|c|}{$\begin{array}{c}x^{5}-30468740 x^{4}-238176040400 x^{3}+7407835961593805 x^{2} \\
+409125920 x-1 \\
\end{array}$} \\
\hline$\overline{8581}$ & 11 & 6 & 1 & 6 & $\overline{1}$ \\
\hline 8641 & & 17 & & 11 & \\
\hline $651=4$ & & 212,165 & $(1,1)$ & 212 & \\
\hline & & 95530 & + & & \\
\hline
\end{tabular}




\begin{tabular}{|c|c|c|c|c|c|}
\hline$f$ & $k$ & $\left(g_{i}\right)_{i=1, \ldots, k}$ & $k-u p l e$ & gen & $h$ \\
\hline \multicolumn{6}{|c|}{$p(x)$} \\
\hline $8651=41.211$ & 2 & 212,165 & $\overline{(2,1)}$ & 212 & $\overline{\overline{5}}$ \\
\hline \multicolumn{6}{|c|}{$\begin{array}{c}x^{5}+3123337663536767 x^{4}-530895782873199374755299 x^{3} \\
-27657890792207426765148205 x^{2}+2236526003587140186 x-1 \\
\end{array}$} \\
\hline $8651=41.211$ & 2 & 212,165 & $\overline{(3,1)}$ & 212 & 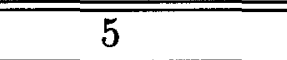 \\
\hline \multicolumn{6}{|c|}{$\begin{array}{c}x^{5}-1849722392220488995516724181572391731821801 x^{4} \\
-40257502738553193737486568666792044421140835168709689498 x^{3} \\
+527259032377192443917552472121253734413506118810949098 x^{2} \\
+71179067779754374837250586830415042331797401 x-1 \\
\end{array}$} \\
\hline $8651=41.211$ & 2 & 212,165 & $(4,1)$ & 212 & $155=5.31$ \\
\hline \multicolumn{6}{|c|}{$x^{5}-1784 x^{4}+101717 x^{3}-186812 x^{2}-3312 x-1$} \\
\hline 8681 & 1 & 15 & 1 & 15 & 1 \\
\hline $8711=31.281$ & 2 & 282,94 & $(1,1)$ & 282 & $55=5.11$ \\
\hline \multicolumn{6}{|c|}{$\begin{array}{l}x^{5}-1653705456782775171912673 x^{4} \\
+5445240764167 x-1 \\
\end{array}$} \\
\hline $8711=31.281$ & 2 & 282,94 & $(2,1)$ & 282 & 5 \\
\hline \multicolumn{6}{|c|}{$\begin{array}{c}x^{5}-915961876093 x^{4}+2020418282662546716107 x^{3} \\
+32075034172803212 x^{2}+21455354497 x-1 \\
\end{array}$} \\
\hline $8711=31.281$ & 2 & 282,94 & $\overline{(3,1)}$ & 282 & $\overline{5}$ \\
\hline \multicolumn{6}{|c|}{$\begin{aligned} & x^{5}+236170027804 x^{4}+302854669123206 x^{3}-1917783429 x^{2} \\
&-178232 x-1 \\
&\end{aligned}$} \\
\hline $8711=31.281$ & 2 & 282,94 & $\overline{(4,1)}$ & 282 & 5 \\
\hline \multicolumn{6}{|c|}{$\begin{array}{r}x^{5}-522450369775896 x^{4}+1587932670649366419698 x^{3} \\
+4682448357713305310743 x^{2}+210177440161096766 x-1 \\
\end{array}$} \\
\hline 8731 & 1 & 2 & 1 & 2 & 1 \\
\hline 8741 & 1 & 2 & 1 & 2 & 1 \\
\hline 8761 & 1 & 23 & 1 & 23 & 1 \\
\hline 8821 & 1 & 2 & 1 & 2 & 1 \\
\hline 8831 & 1 & 7 & 1 & 7 & 16 \\
\hline \multicolumn{6}{|c|}{$\begin{array}{c}x^{5}-351955773522719 x^{4}-2448906828520064103847890434 x^{3} \\
+21060722808988447787381920060918946 x^{2} \\
-1650384503959872998714936366251585 x+1 \\
\end{array}$} \\
\hline 8861 & 1 & 2 & 1 & 2 & 1 \\
\hline $8921=11.811$ & 2 & 1623,23 & $(1,1)$ & 1623 & 5 \\
\hline \multicolumn{6}{|c|}{$\begin{array}{c}x^{5}-45628488555 x^{4}+2391819282026291135 x^{3} \\
+15517482843586408 x^{2}+1621369115 x-1 \\
\end{array}$} \\
\hline
\end{tabular}




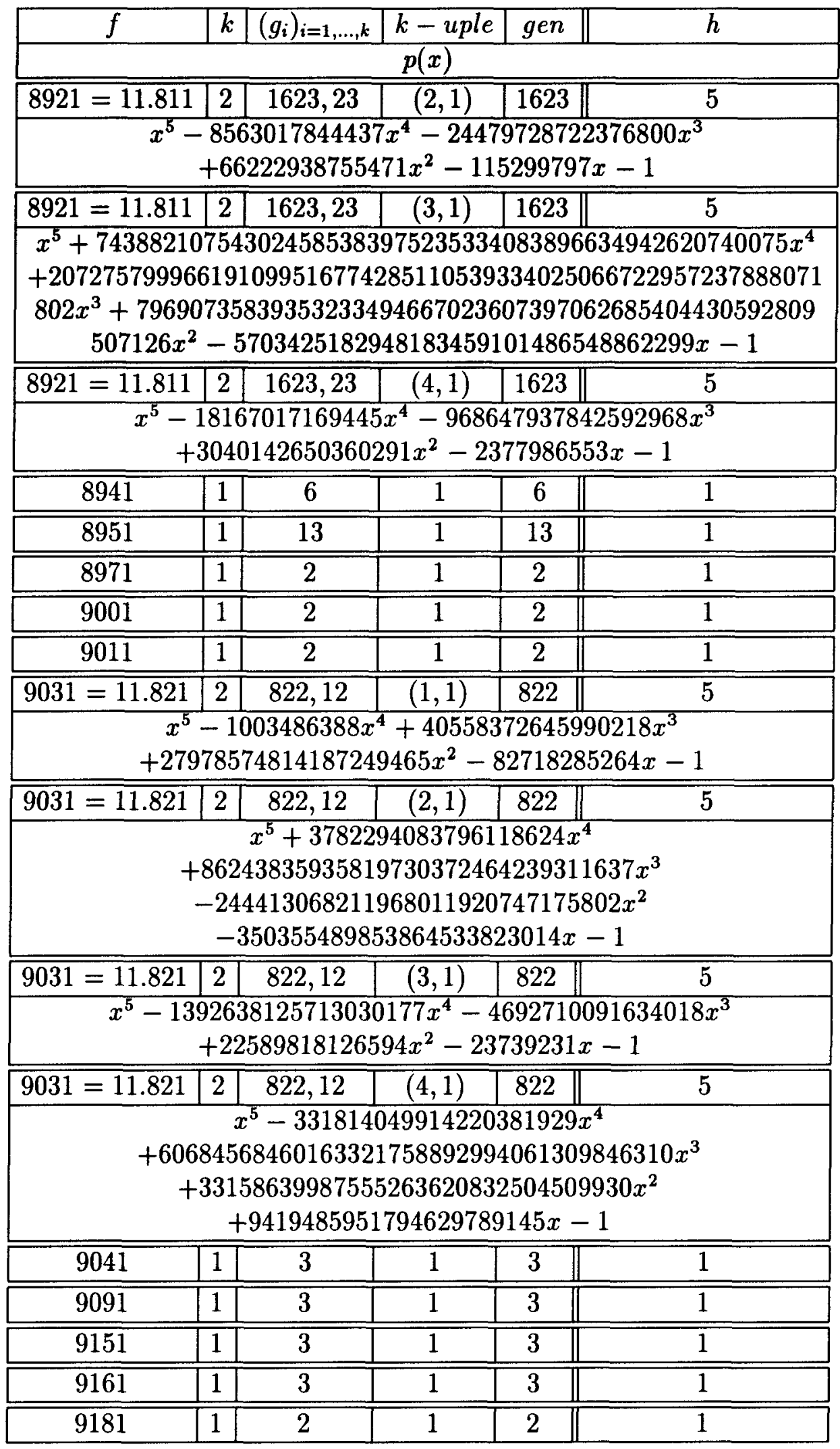




\begin{tabular}{|c|c|c|c|c|c|}
\hline $\bar{f}$ & & $\left(g_{i}\right)_{i=1, \ldots, k}$ & $k-u p l e$ & gen & $h$ \\
\hline \multicolumn{6}{|c|}{$p(x)$} \\
\hline $9211=61.151$ & \begin{tabular}{|l|}
2 \\
\end{tabular} & 152,428 & $(1,1)$ & $\mid$\begin{tabular}{|l||}
152 \\
\end{tabular} & $\overline{5}$ \\
\hline \multicolumn{6}{|c|}{$\begin{aligned} x^{5}+542851643831 x^{4} & +1289935953145 x^{3}-13949894688 x^{2} \\
& -240701 x-1\end{aligned}$} \\
\hline $9211=61.151$ & 2 & 152,428 & $\overline{(2,1)}$ & $|152| \mid$ & $\overline{5}$ \\
\hline \multicolumn{6}{|c|}{$\begin{array}{l}x^{5}-1138014236 x^{4}-9522161817838885 x^{3} \\
+6036276062610202 x^{2}-22324397952 x-1\end{array}$} \\
\hline $9211=61.151$ & \begin{tabular}{|l|l}
2 & \\
\end{tabular} & $\overline{c 152,428}$ & $\overline{(\overline{(3,1)}}$ & \begin{tabular}{|l||}
152 \\
\end{tabular} & $\overline{5}$ \\
\hline \multicolumn{6}{|c|}{$\begin{aligned} x^{5}+138653685208 x^{4} & +1433445654317 x^{3}-1349764346763 x^{2} \\
& +37211225 x-1\end{aligned}$} \\
\hline $9211=61.151$ & \begin{tabular}{|l|l}
2 & \\
\end{tabular} & (152,428 & (4,1) & \begin{tabular}{|l||}
152 \\
\end{tabular} & $\overline{\overline{5}}$ \\
\hline \multicolumn{6}{|c|}{$\begin{array}{r}x^{5}+15775075131618 x^{4}+56988196368888931229234 x^{3} \\
-588071618199094594233743777 x^{2}-26477283380736000 x-1 \\
\end{array}$} \\
\hline$\overline{9221}$ & \begin{tabular}{l|l}
1 & \\
\end{tabular} & $\overline{\overline{2}}$ & $\overline{11}$ & $\overline{2}$ & $\overline{11}$ \\
\hline 9281 & 11 & $\overline{3}$ & $\overline{11}$ & $\overline{3}$ & $\overline{1}$ \\
\hline$\overline{9311}$ & $\overline{11}$ & $\overline{7}$ & $\overline{11}$ & 7 & $\overline{11}$ \\
\hline 9341 & $\overline{11}$ & $\overline{2}$ & $\overline{1}$ & $\overline{2}$ & $\overline{11}$ \\
\hline 9371 & 1 & 2 & 1 & 2 & 1 \\
\hline 9391 & \begin{tabular}{|l|l|}
1 \\
\end{tabular} & $\overline{3}$ & $\overline{11}$ & $\overline{3}$ & $\overline{1}$ \\
\hline 9421 & \begin{tabular}{|l|l|}
1 & \\
\end{tabular} & 2 & 1 & 2 & 11 \\
\hline \multicolumn{6}{|c|}{$\begin{array}{c}x^{5}-9007815260 x^{4}+17253222687758533 x^{3} \\
+692307848252787734521 x^{2}-34800965611636050725 x+1 \\
\end{array}$} \\
\hline$\overline{9431}$ & \begin{tabular}{|l|l|}
1 & \\
\end{tabular} & $\overline{7}$ & $\overline{11}$ & $\overline{7}$ & $\overline{1}$ \\
\hline$\overline{9461}$ & \begin{tabular}{|l|l|}
1 & \\
\end{tabular} & $\overline{3}$ & $\overline{1}$ & $\overline{3}$ & $\overline{1}$ \\
\hline 9491 & \begin{tabular}{|l|l|l|}
1 & \\
\end{tabular} & 2 & 1 & 2 & 1 \\
\hline$\overline{9511}$ & \begin{tabular}{l|l}
1 & \\
\end{tabular} & $\overline{3}$ & $\overline{1}$ & $\overline{3}$ & $\overline{1}$ \\
\hline$\overline{9521}$ & \begin{tabular}{l|l}
1 & \\
\end{tabular} & $\overline{3}$ & $\overline{1}$ & $\overline{3}$ & 1 \\
\hline$\overline{9551}$ & \begin{tabular}{l|l}
1 &
\end{tabular} & 11 & $\overline{11}$ & 11 & 541 \\
\hline \multicolumn{6}{|c|}{$x^{5}+9354 x^{4}-95268 x^{3}-84065 x^{2}-1018 x+1$} \\
\hline$\overline{\overline{9601}}$ & $1 \mid$ & $\overline{13}$ & $\overline{\overline{1}}$ & 13 & 1 \\
\hline$\overline{9631}$ & \begin{tabular}{l|l}
1 & \\
\end{tabular} & $\overline{3}$ & $\overline{1}$ & $\overline{3}$ & $\overline{1}$ \\
\hline $9641=31.311$ & \begin{tabular}{l|l}
2 & \\
\end{tabular} & 623,342 & $(1,1)$ & 623 & 5 \\
\hline \multicolumn{6}{|c|}{$\begin{array}{c}x^{5}+1990147499262615809371644065631 x^{4} \\
-7094657869877186941746657730668637626642818 x^{3} \\
07563629572118097867827450018060457866851496445858 x^{2} \\
-14979217332111212775979898715692927 x-1\end{array}$} \\
\hline
\end{tabular}




\begin{tabular}{|c|c|c|c|c|c|}
\hline$f$ & $k$ & $\left(g_{i}\right)_{i=1, \ldots, k}$ & $k-u p l e$ & gen & $h$ \\
\hline \multicolumn{6}{|c|}{$p(x)$} \\
\hline $9641=31.311$ & 2 & 623,342 & $\overline{(\overline{(2,1)}}$ & "623 & $\overline{5}$ \\
\hline \multicolumn{6}{|c|}{$\begin{array}{l}x^{5}-19278890062149 x^{4}-22392929195953446 x^{3} \\
+419824576200873445 x^{2}+36385068409 x-1 \\
\end{array}$} \\
\hline $9641=31.311$ & 2 & 623,342 & $\overline{(3,1)}$ & \begin{tabular}{|c|}
623 \\
\end{tabular} & $\overline{5}$ \\
\hline \multicolumn{6}{|c|}{$\begin{array}{c}x^{5}-883348642453707085 x^{4}-23413567478952827177 x^{3} \\
-17357028503300766904 x^{2}-887965609205 x-1\end{array}$} \\
\hline $9641=31.311$ & \begin{tabular}{|l|}
2 \\
\end{tabular} & 623,342 & $\overline{(4,1)}$ & \begin{tabular}{ll|}
623 \\
\end{tabular} & $\overline{5}$ \\
\hline \multicolumn{6}{|c|}{$\begin{aligned} x^{5} & +57223906751 x^{4}-97247824699481252481 x^{3} \\
& +8104733900491400 x^{2}-476253863 x-1\end{aligned}$} \\
\hline 9661 & 1 & 2 & 1 & 2 & 1 \\
\hline $9691=11.881$ & 2 & 882,12 & $(1,1)$ & 882 & 5 \\
\hline \multicolumn{6}{|c|}{$\begin{array}{l}x^{5}+2029865420310798556371 x^{4} \\
-7344104554136018217119966737 x^{3} \\
78971367663905159 x^{2}+371033034193784509728 x-1 \\
\end{array}$} \\
\hline $9691=11.881$ & 2 & 882,12 & $(2,1)$ & 882 & 5 \\
\hline \multicolumn{6}{|c|}{$\begin{array}{l}x^{5}+1359252153 x^{4}+6549311934124112 x^{3} \\
+90994858794255659 x^{2}+1102977309 x-1 \\
\end{array}$} \\
\hline $9691=11.881$ & $2 \mid$ & $\overline{882,12}$ & $\overline{(3,1)}$ & 882 & $\overline{5}$ \\
\hline \multicolumn{6}{|c|}{$\begin{array}{c}x^{5}-20519119145738603111972635529941 x^{4} \\
-108471003760997615733269641534207608396693810656646 x^{3} \\
-77743176232756598404512775609410515870043466890 x^{2} \\
-68930664135170696469110973706758619 x-1\end{array}$} \\
\hline $9691=11.881$ & 2 & 882,12 & $(4,1)$ & 882 & 5 \\
\hline \multicolumn{6}{|c|}{$\begin{array}{r}x^{5}+1030589581917829709 x^{4}+76061587548778237 x^{3} \\
+15975341887308 x^{2}+167039911 x-1\end{array}$} \\
\hline 9721 & 1 & 7 & 1 & 7 & 1 \\
\hline 9781 & 1 & $\overline{6}$ & 1 & $\overline{6}$ & 1 \\
\hline 9791 & 1 & 11 & 1 & 11 & 1 \\
\hline 9811 & 1 & 3 & 1 & 3 & 1 \\
\hline 9851 & 1 & 2 & 1 & 2 & 1 \\
\hline 9871 & 1 & 3 & 1 & 3 & 1 \\
\hline $9881=41.241$ & 21 & 965,42 & $(1,1)$ & 965 & $155=5.31$ \\
\hline \multicolumn{6}{|c|}{$x^{5}-490 x^{4}-12651 x^{3}-24970 x^{2}+18048 x-1$} \\
\hline $9881=41.241$ & 2 & 965,42 & $(2,1)$ & 965 & 5 \\
\hline \multicolumn{6}{|c|}{$\begin{array}{c}x^{5}-581265 x^{4}+29555449667 x^{3}+2390046222485999 x^{2} \\
+7403616408 x-1\end{array}$} \\
\hline
\end{tabular}




\begin{tabular}{|c|c|c|c|c||c|}
\hline$f$ & $k$ & $\left(g_{i}\right)_{i=1, \ldots, k}$ & $k$-uple & gen & $h$ \\
\hline \multicolumn{5}{|c|}{$p(x)$} \\
\hline \hline $9881=41.241$ & 2 & 965,42 & $(3,1)$ & 965 & 5 \\
\hline$x^{5}+559440637634965 x^{4}+10855688297025590117 x^{3}$ \\
$+5053023034057664860991 x^{2}+4212393258961459752 x-1$ \\
\hline \hline $9881=41.241$ & 2 & 965,42 & $(4,1)$ & 965 & 5 \\
\hline$x^{5}+421120053 x^{4}+123226803043838 x^{3}$ \\
$+57657577123201 x^{2}-6549250341653 x-1$ \\
\hline \hline 9901 & 1 & 2 & 1 & 2 & 1 \\
\hline \hline 9931 & 1 & 10 & 1 & 10 & 1 \\
\hline \hline 9941 & 1 & 2 & 1 & 2 & 1 \\
\hline
\end{tabular}

\section{Références}

[G] G.Gras et M.-N.Gras, "Calcul du nombre de classes et des unités des extensions abéliennes réelles de $\mathbb{Q}$ ", Bull. Sci. Math. , V. 101 (1977), pp. 97-129.

[GG] G.Gras, "GALCYCL", Publ. Math. Fac. sci. Besançon (Théorie des nombres), année $84 / 85$ et $85 / 86$, fascicule 2 .

Stéphane Jeannin

UFR Sciences et Techniques

Laboratoire de Mathématiques

URA 741 au CNRS

F-25030 Besançon Cédex. 